\title{
AdS vacua with scale separation from IIB supergravity
}

\section{Petrini, ${ }^{a}$ G. Solard ${ }^{a}$ and T. Van Riet ${ }^{b}$}

${ }^{a}$ Laboratoire de Physique Théorique et Hautes Energies, Université Pierre et Marie Curie, 4 Place Jussieu, 75252 Paris Cedex 05, France

${ }^{b}$ Instituut voor Theoretische Fysica, K.U. Leuven, Celestijnenlaan 200D, B-3001 Leuven, Belgium

E-mail: petrini@lpthe.jussieu.fr, solard@lpthe.jussieu.fr, thomasvr @ itf.fys.kuleuven.be

ABSTRACT: Only two kinds of compactification are known that lead to four-dimensional supersymmetric AdS vacua with moduli stabilisation and separation of scales at tree-level. The most studied ones are compactifications of massive IIA supergravity on SU(3) structures with smeared O6 planes, for which a general ten-dimensional expression for the solution in terms of the $\mathrm{SU}(3)$ structure was found. Less studied are compactifications of IIB supergravity with smeared $05 / \mathrm{O} 7$ planes. In this paper we derive a general tendimensional expression for the smeared $\mathrm{O} 5 / \mathrm{O} 7$ solutions in terms of $\mathrm{SU}(2)$ structures. For a specific choice of orientifold projections, we recover the known examples and we also provide new explicit solutions.

KeYwords: Flux compactifications, Differential and Algebraic Geometry, Supergravity Models, Superstring Vacua

ARXiv EPRINT: 1308.1265 


\section{Contents}

1 Introduction 2

2 Type IIB $\mathrm{AdS}_{4}$ vacua with $\mathcal{N}=1$ supersymmetry 4

2.1 General constraints 6

$\begin{array}{lll}2.2 & \text { Supersymmetry and SU(2) torsion classes } & 7\end{array}$

$3 \quad$ A simple class of $\mathrm{SU}(2)$ structure geometries $\quad 9$

4 Scale separation $\quad \mathbf{1 2}$

$\begin{array}{ll}\text { 4.1 Separation of scales without sources? } & 15\end{array}$

5 Explicit examples $\quad 16$

$\begin{array}{llr}5.1 \text { Coset manifolds } & 19\end{array}$

$\begin{array}{lll}5.1 .1 & \frac{\mathrm{SU}(3) \times \mathrm{U}(1)}{\mathrm{SU}(2)} & 20\end{array}$

$\begin{array}{ll}5.1 .2 \frac{\mathrm{SU}(2)^{2}}{\mathrm{U}(1)} \times \mathrm{U}(1) & 21\end{array}$

5.1.3 $\quad \mathrm{SU}(2) \times \mathrm{SU}(2)$ and $\mathrm{SU}(2) \times \mathrm{U}(1)^{3} \quad 22$

5.2 Nilmanifolds 22

5.2.1 Model 3.14 23

$\begin{array}{lll}5.2 .2 & \text { Model } 4.1 & 23\end{array}$

5.2.3 Model $5.1 \quad 24$

6 Discussion $\quad 25$

A Conditions for $\mathrm{AdS}_{4}$ vacua with $\mathcal{N}=1$ SUSY in type IIB SUGRA 26

$\begin{array}{lll}\text { A.1 } & \mathrm{SU}(3) \text { and } \mathrm{SU}(2) \text { structures } & 26\end{array}$

$\begin{array}{ll}\text { A.1.1 Torsion classes } & 28\end{array}$

$\begin{array}{ll}\text { A.2 Conditions for } \mathrm{AdS}_{4} \text { vacua } & 28\end{array}$

A.3 No $\mathrm{AdS}_{4}$ vacua for $\mathrm{SU}(3)$ structure 30

$\begin{array}{lll}\text { A.4 } & \mathrm{AdS}_{4} \text { vacua for } \mathrm{SU}(2) \text { structures } & 30\end{array}$

$\begin{array}{lll}\text { A.4.1 Rigid SU(2) structures } & 30\end{array}$

B Type IIA $\mathbf{A d S}_{4}$ solutions and separation of scales 33

B.1 Lust-Tsimpis SU(3)-structures 33

$\begin{array}{lll}\text { B.2 The rectangular torus solution } & 35\end{array}$

C Computation of the 6D Ricci scalar $\quad 36$ 


\section{Introduction}

One of the ultimate goals of the research on flux compactifications is the construction of flux vacua that break supersymmetry in a solution with a small positive cosmological constant. Despite many interesting ideas and proposals, it is still debatable whether there exist fully explicit and controllable solutions to this problem. A possible strategy is to break up the problem in parts and solve each part separately. In this paper we therefore settle with studying SUSY AdS vacua and set aside the issue of SUSY breaking and positive cosmological constant. Despite the fact that SUSY AdS vacua are the best known and most constructed solutions in string theory, there are still some obvious and interesting problems as we point out below.

Before the original KKLT proposal [1] (and [2]), none of the AdS vacua in string theory were truly lower-dimensional in the sense that the AdS scale was not parametrically larger than the length scale of the extra dimensions. This is the most straightforward constraint that observations put on flux vacua. The AdS solutions that are used for holography typically do not have scale separation and it is important to understand how holography works for AdS vacua with scale separation [3]. The KKLT construction and its descendants are not entirely explicit from a 10-dimensional point of view, which complicates a possible holographic understanding. For that reason, and for reasons of elegance and simplicity, it would be desirable to have solutions of classical supergravity in ten dimensions. This was first claimed in a series of papers constructing such vacua in massive IIA supergravity with intersecting O6 planes [4-6] (see also [7, 8] for later work on these solutions). In massive IIA many solutions without sources are also known $[9,10]$, but they cannot achieve scale separation [11]. Only for solutions with O6 planes is this possible, although no no-go theorem excluding other possibilities has been found.

It is unfortunate that the orientifold and D-brane sources in these compactifications are not fully understood. Most prominent is the fact that in most cases the supergravity solutions are constructed in the limit where the sources are smeared over the transverse space (see for instance the discussion in [12]). The way the solution is supposed to change for fully localised sources is still an open question, even if some interesting progress was made in [13]. For compactifications with sources that are parallel (or have an F-theory interpretation), such as for the no-scale orientifold compactifications of $[14,15]$ and their T-duals [16-18], it is known how to treat fully localised sources. For these cases the backreaction does not invalidate the existence of the solutions, but it is expected to be very relevant when computing fluctuations around the vacuum (see for instance [19-23]). However the AdS compactifications in IIA with scale separation involve intersecting O6 planes and, apart from the partial results in [13], not much is known. Another issue that troubles these vacua is more stringy and concerns the proper definition of string theory with O6 planes when there is non-zero Romans mass $[12,24]$. Since there is no conventional lift of massive IIA supergravity to 11 dimensions ${ }^{1}$ it is not clear how the orientifold singularities can be resolved and whether the background makes sense. ${ }^{2}$

\footnotetext{
${ }^{1}$ See however the intriguing proposal of [25], or the alternative suggestion that a lift is unnecessary since massive IIA cannot be strongly coupled at weak curvature [26].

${ }^{2}$ See reference [27] for more radical doubts about the use of orientifold planes.
} 
For the reasons just named it is relevant to find other classical AdS solutions with scale separation in a different context. In this paper we consider type IIB supergravity (string theory). It is commonly claimed that this theory cannot achieve moduli stabilisation at the classical level, but this statement can readily be violated by considering non-geometric fluxes or by moving beyond the usual O3/O7 compactifications and instead relying on O5/O7 orientifolds. A first attempt at finding such vacua has been done in [28], where the authors considered four dimensional effective theories obtained by consistent truncations on specific $\mathrm{SU}(2)$ structure manifolds (built from coset space coverings) with smeared O5/O7 intersections. While some of the models considered allow for full moduli stabilisation, it is not clear whether they admit a limit in which the solution is at large volume, weak coupling and with scale separation.

The aim of this paper is to further study O5/O7 compactifications of IIB supergravity to four-dimensional, unwarped AdS space, the absence of warping being a necessary outcome of the approximation of smeared sources. Our results have partial overlap with an earlier investigation on SUSY AdS vacua in IIA/IIB SUGRA [29]. We construct the solutions directly in ten dimensions using the pure spinors approach proposed in $[16,30,31]$.

For compactifications to four dimensions, this formalism allows to reduce the study of ten-dimensional supersymmetric backgrounds to the analysis of a set of equations involving only the components of the fields on the internal manifold. In this case, it is easy to show that the O-plane projections and supersymmetry require the internal manifold to admit a rigid $\mathrm{SU}(2)$ structure. It is then possible to write down a general solution for the fields on the compactification manifold. By general solution we mean a set of constraints on the six-dimensional fields that are applicable to a whole class of manifolds instead of a specific example. This is typically achieved through writing the solution in terms of the SU(2) invariant forms on the manifolds. To go from this general form to a concrete example one only has to compute the canonical forms for a given manifold. This is clearly beneficial and more insightful than minimizing $F$ and $D$ terms for a given manifold. When the compactification manifolds allow for consistent truncations, which is the case for homogeneous manifolds with smeared sources, then the minima of the scalar potential must lift consistently to solutions of the equations of motion in ten dimensions, such as derived in this paper. Reference [32] explicitly analysed how the IIA vacua in 4D lift to 10 IIA SUGRA solutions with smeared sources.

Our analysis parallels the derivation given in [16] of the conditions for $\mathcal{N}=1 \mathrm{AdS}_{4}$ vacua of [7]. However the IIB case seems to have a much richer spectrum of solutions than the IIA case. For this reason, in looking for explicit examples, we will restrict to homogeneous spaces. We perform a systematic scan of the coset manifolds of [33] and nilmanifolds. Although we have not been able to find solutions fulfilling the criteria of weak coupling and scale separation on the cosets, we have found such solutions on nilmanifolds thereby extending the single solution that was known so far [8], which was obtained by T-dualising the O6 solution on the six-torus. Although these new solutions are also incomplete in the sense that the sources are smeared, they constitute an important step since they change the existing paradigm that tree-level scale separation is only possible in massive IIA. Besides providing more examples of tree-level scale separation, the examples in IIB could be 
relevant for improving our understanding of the subtleties in such backgrounds, such as the orientifold singularities. The usual criticism of using O6 planes in the presence of Romans mass is clearly evaded here.

The paper is organised as follows. In section 2 we give the general conditions that the fields on the internal manifold have to satisfy in order to have $\mathcal{N}=1 \mathrm{AdS}_{4}$ with $\mathrm{O} 5 / \mathrm{O} 7$ planes. Since the derivation is quite lengthy we put it in appendix A. In section 3 we specify the general system to a class of $\mathrm{SU}(2)$ structures that are for instance allowed on homogeneous spaces. The orientifold projections and the restriction to left-invariant forms make it possible to solve explicitly for most of the constraints. In section 4 we discuss possible criteria to check whether a given vacuum admit separation of scales. Finally in section 5 we present an exhaustive list of $\mathcal{N}=1 \mathrm{AdS}_{4}$ with $\mathrm{O} 5 / \mathrm{O} 7$ planes that can be found on cosets and nilmanifolds and we discuss their properties as good 4-dimensional vacua.

Appendix A contains the definitions of $\mathrm{SU}(3)$ and $\mathrm{SU}(2)$ structures and pure spinors we need in the rest of the paper, and the derivation of the general conditions for $\mathcal{N}=1$ $\mathrm{AdS}_{4}$ susy vacua. For completeness in appendix $\mathrm{B}$ we give the form of the generic $\mathcal{N}=1$ $\mathrm{AdS}_{4}$ vacuum in type IIA and discuss an example of separation of scale in this context. Finally, in appendix $\mathrm{C}$ we detail the form of the Ricci scalar for the class of SU(2) solutions we consider in this paper.

\section{Type IIB $\mathrm{AdS}_{4}$ vacua with $\mathcal{N}=1$ supersymmetry}

A standard technique to study supersymmetric vacua is to look for solutions to the supersymmetry variations plus the Bianchi identities for the fluxes. In presence of non-trivial backgrounds fluxes, instead of working directly with spinorial equations, it is more convenient to rewrite the susy equations as a set of differential conditions on forms. This is the idea behind the application of $G$-structures and more generally Generalised Complex Geometry [34, 35]. We begin this section with a brief overview of the formalism we need to determine our solutions. Details can be found in appendix A.

In the Generalised Complex Geometry approach the main ingredients are a pair of polyforms, $\Phi_{ \pm}$, which are constructed as bilinears in the supersymmetry parameters on the internal manifold $Y$

$$
\Phi_{ \pm}=\eta_{+}^{1} \otimes \eta_{ \pm}^{2 \dagger}
$$

where $\eta_{+}^{i}$ are six-dimensional Weyl spinors, and $\eta_{-}^{i}=\left(\eta_{+}^{i}\right)^{*}$. Then, the ten-dimensional supersymmetry variations can be rewritten as a set of differential conditions on such forms. For Type IIB compactifications to $\mathrm{AdS}_{4}$, the susy conditions are [30]

$$
\begin{aligned}
(\mathrm{d}-H \wedge)\left(e^{2 A-\phi} \Phi_{-}\right) & =-2 \mu e^{A-\phi} \operatorname{Re} \Phi_{+}, \\
(\mathrm{d}-H \wedge)\left(e^{A-\phi} \operatorname{Re} \Phi_{+}\right) & =0 \\
(\mathrm{~d}-H \wedge)\left(e^{3 A-\phi} \operatorname{Im} \Phi_{+}\right) & =-3 e^{2 A-\phi} \operatorname{Im}\left(\bar{\mu} \Phi_{-}\right)-\frac{1}{8} e^{4 A} * \lambda(F),
\end{aligned}
$$

where $\phi$ is the dilaton, $A$ the warp factor

$$
\mathrm{d} s^{2}=e^{2 A} \mathrm{~d} s_{(4)}^{2}+\mathrm{d} s_{(6)}^{2},
$$


and $F$ is the sum of the RR field strength on $Y, F=F_{1}+F_{3}+F_{5} . \lambda$ acts on a form as the transposition of all indices

$$
\lambda\left(F_{k}\right)=(-)^{[k / 2]} F_{k} .
$$

The ten-dimensional fluxes are defined in terms of $F$ by

$$
F_{(10)}=\operatorname{vol}_{4} \wedge \lambda(* F)+F .
$$

Finally, the complex number $\mu$ determines the size of the $\mathrm{AdS}_{4}$ cosmological constant

$$
\Lambda=-|\mu|^{2} .
$$

The form of the pure spinors $\Phi_{ \pm}$depends on the relation between the internal spinors $\eta_{1}$ and $\eta_{2}$. In the most general case we have

$$
\begin{aligned}
& \Phi_{-}=-\frac{a b}{8} z \wedge\left(k_{\perp} e^{-i j}+i k_{\|} \omega\right), \\
& \Phi_{+}=\frac{a \bar{b}}{8} e^{z \bar{z} / 2}\left(k_{\|} e^{-i j}-i k_{\perp} \omega\right),
\end{aligned}
$$

where $z, j$ and $\omega$ are a one-form, a real two-form and a holomorphic two-form, respectively, which are globally defined on the internal manifold and define a SU(2) structure. ${ }^{3}$ The complex functions $a$ and $b$ are related to the norms of the spinors $\eta^{i}$

$$
\left\|\eta_{+}^{1}\right\|^{2}=|a|^{2}, \quad\left\|\eta_{+}^{2}\right\|^{2}=|b|^{2},
$$

and to the norm of the pure spinors

$$
\left\langle\Phi_{ \pm}, \bar{\Phi}_{ \pm}\right\rangle=-i\left\|\Phi_{ \pm}\right\|^{2} \operatorname{vol}_{6}=-\frac{i}{8}|a|^{2}|b|^{2} \operatorname{vol}_{6},
$$

where vol $_{6}$ is the volume of the internal manifold and the product

$$
\langle A, B\rangle=\left.(A \wedge \lambda(B))\right|_{\text {top }}
$$

is the Mukai pairing among forms.

When $k_{\|}=1$ and $k_{\perp}=0$ the spinors $\eta_{+}^{1}$ and $\eta_{+}^{2}$ are parallel and the internal manifold is said to be of $\mathrm{SU}(3)$ structure. The pure spinors reduce to

$$
\begin{aligned}
& \Phi_{-}=-i \frac{a b}{8} \Omega, \\
& \Phi_{+}=\frac{a \bar{b}}{8} e^{-i J},
\end{aligned}
$$

where $\Omega$ and $J$ are the $\mathrm{SU}(3)$ invariant forms defining the $\mathrm{SU}(3)$ structure, (A.4). In the opposite case, $k_{\|}=0$ and $k_{\perp}=1$, the spinors $\eta_{+}^{1}$ and $\eta_{+}^{2}$ are orthogonal and the structure is $\mathrm{SU}(2)$ with

$$
\begin{aligned}
& \Phi_{-}=-\frac{a b}{8} z \wedge e^{-i j}, \\
& \Phi_{+}=-i \frac{a \bar{b}}{8} e^{z \bar{z} / 2} \omega .
\end{aligned}
$$

\footnotetext{
${ }^{3}$ The definition of $\mathrm{SU}(2)$ and $\mathrm{SU}(3)$ structures can be found in appendix A.
} 
The general case, where the relative orientation of $\eta_{+}^{1}$ an $\eta_{+}^{2}$ can vary on the manifold is often referred to as dynamical $\mathrm{SU}(2)$ structure.

For AdS vacua, supersymmetry constraints the norms of the two six-dimensional spinors to be equal [16]

$$
|a|^{2}=|b|^{2}=e^{A}
$$

Only the relative scale between the spinor being relevant, we can always rescale $\eta_{+}$in such a way that

$$
\bar{b}=a, \quad \frac{b}{a}=e^{-i \theta} .
$$

Equation (2.4) can be seen as a definition of the RR fluxes that are compatible with $\mathcal{N}=1$ supersymmetry. In order to have a full solution of the ten-dimensional equations of motion, one must also check that the RR fluxes determined this way satisfy the Bianchi identities

$$
\begin{aligned}
\mathrm{d} H & =0, \\
\mathrm{~d} F-H \wedge F & =\delta(\text { sources }),
\end{aligned}
$$

where $\delta$ (sources) denotes the charge density of the space-filling sources. In this paper we will mostly consider space-filling O5 and O7-planes intersecting on the internal manifold. Since we do not know how to find exact solutions that describe generic intersecting branes or O-planes, we smear them over the internal manifold, and we write the source terms as invariant smooth forms on the internal manifold ${ }^{4}$

$$
\mathrm{d} F-H \wedge F=\sum_{i} c_{i} \alpha^{i}=\sum_{i} Q_{i}(\text { source }) \operatorname{vol}_{i}
$$

where $c_{i}$ are constants and $Q_{i}$ is the charge density of the source. The symbol $\alpha^{i}$ denote a decomposable form dual to the cycle wrapped by the brane, while $\operatorname{vol}_{i}$ is the volume form dual to the cycle.

\subsection{General constraints}

Plugging the expression (2.14) of the pure spinors in the SUSY equations, it is easy to see that, for $\mathrm{SU}(3)$ structure manifolds, (2.2) has no solutions. We recover the known result that there are no $\mathcal{N}=1 \mathrm{AdS}_{4}$ vacua with $\mathrm{SU}(3)$ structure in type IIB supergravity [37].

We are left with the possibility of rigid or dynamical SU(2) structure. By expanding the supersymmetry equations in forms of definite degree we can package the conditions for $\mathrm{AdS}_{4}$ vacua with $\mathcal{N}=1$ supersymmetry as a set of differential constraints on the $\mathrm{SU}(2)$ structure forms, the fluxes and the functions $k_{\|}$and $k_{\perp}$. Let us consider again (2.2). The zero-form component

$$
\mu k_{\|} \cos \theta=0
$$

\footnotetext{
${ }^{4}$ We refer to appendix $\mathrm{C}$ of [8] for an explanation on smeared source terms and the corresponding microscopic interpretation in terms of orientifolds and their involutions, whereas appendix D of [36] contains some first attempts for charge and flux quantisation.
} 
gives a constraint on the parameters $k_{\|}$and $\theta_{-}$, since for AdS vacua $\mu \neq 0$. Choosing

$$
\cos \theta=0,
$$

fixes the relative phase of the spinors $\eta^{1}$ and $\eta^{2}$

$$
\theta=\theta_{a}-\theta_{b}=\frac{\pi}{2} \quad \Rightarrow \quad a=i b .
$$

Such phase is related to the choice of orientifold planes that one can add as sources, see for instance [16, 38]. On can show that $a=i b$ is compatible with O7-planes only. Since we want to be free to have also O5-planes, we are forced to choose the first option and set

$$
k_{\|}=0 \quad k_{\perp}=1,
$$

which means that we will only consider backgrounds of rigid SU(2)-structure. For this case the supersymmetry conditions reduce to a set of equations for the $\mathrm{SU}(2)$ structure forms (see again appendix A for the derivation)

$$
\begin{aligned}
\mathrm{d}\left(e^{3 A-\phi} z\right) & =2 \mu e^{2 A-\phi} \operatorname{Im} \hat{\omega}, \\
z \wedge\left(\mathrm{d} j-i H+\mu e^{-A} \bar{z} \operatorname{Re} \hat{\omega}\right) & =0 \\
\mathrm{~d}\left(e^{2 A-\phi} \operatorname{Im} \hat{\omega}\right) & =0, \\
\mathrm{~d}\left(e^{2 A-\phi} z \wedge \bar{z} \wedge \operatorname{Re} \hat{\omega}\right) & =2 i e^{2 A-\phi} H \wedge \operatorname{Im} \hat{\omega},
\end{aligned}
$$

and the following equations for the RR fluxes

$$
\begin{aligned}
* F_{5}= & -3 e^{-A-\phi} \operatorname{Im}(\bar{\mu} z), \\
* F_{3}= & -e^{-4 A} \mathrm{~d}\left(e^{4 A-\phi} \operatorname{Re} \hat{\omega}\right)-3 e^{-A-\phi} \operatorname{Re}(\bar{\mu} z) \wedge j, \\
* F_{1}= & i \mathrm{~d}(2 A-\phi) z \wedge \bar{z} \wedge \operatorname{Im} \hat{\omega}+e^{-\phi} H \wedge \operatorname{Re} \hat{\omega} \\
& -\frac{1}{2} e^{-A-\phi} \operatorname{Im}(\bar{\mu} z) \wedge j \wedge j .
\end{aligned}
$$

To simplify the notation, we defined the form $\hat{\omega}=e^{i \theta} \omega$. As mentioned before, in order to find solutions, we have to add to this set of constraints the Bianchi identities for the fluxes.

\subsection{Supersymmetry and $\mathrm{SU}(2)$ torsion classes}

To make contact with previous literature, we can express the equations above in terms of SU(2) torsion classes. The idea here is to decompose all fields in the supersymmetry variations into irreducible representations of the $\mathrm{SU}(2)$ structure group. In general the forms $z, j$ and $\omega$ are not closed. Their deviation from closure can be expressed in terms of different $\mathrm{SU}(2)$ representations, called the torsion classes ${ }^{5}$ [41]

$$
\begin{aligned}
& \mathrm{d} z=S_{1} \omega+S_{2} j+S_{3} z \wedge \bar{z}+S_{4} \bar{\omega}+z \wedge\left(V_{1}+\bar{V}_{2}\right)+\bar{z} \wedge\left(V_{3}+\bar{V}_{4}\right)+T_{1} \\
& \mathrm{~d} j=S_{5} \bar{z} \wedge \omega+S_{6} z \wedge \omega+\frac{1}{2}\left(S_{7}+\bar{S}_{8}\right) z \wedge j+j \wedge V_{5}+z \wedge \bar{z} \wedge V_{6}+z \wedge T_{2}+\text { c.c. }
\end{aligned}
$$

\footnotetext{
${ }^{5}$ See $[39,40]$ for a detailed discussion of intrinsic torsion.
} 


$$
\begin{aligned}
\mathrm{d} \omega= & S_{7} z \wedge \omega+S_{8} \bar{z} \wedge \omega-2 \bar{S}_{5} z \wedge j-2 \bar{S}_{6} \bar{z} \wedge j+i z \wedge \bar{z} \wedge\left(\bar{V}_{6}\llcorner\omega)+j \wedge\left(V_{7}+\bar{V}_{8}\right)\right. \\
& +z \wedge T_{3}+\bar{z} \wedge T_{4} .
\end{aligned}
$$

The coefficients $S_{i}, V_{i}$ and $T_{i}$ denote the 20 different $\mathrm{SU}(2)$ torsion classes: 8 complex singlet $S_{i}, 8$ complex doublets ${ }^{6} V_{i}$ and 4 complex triplets $T_{i}$.

The NS and RR fluxes can also be decomposed according to $\mathrm{SU}(2)$ representations

$$
\begin{aligned}
& H=h_{1} z \wedge \hat{\omega}+h_{2} \bar{z} \wedge \hat{\omega}+h_{3} z \wedge j+z \wedge \bar{z} \wedge h_{1}^{(2)}+h_{2}^{(2)} \wedge j+z \wedge h^{(3)}+\text { c.c. } \\
& F_{1}=f_{1} z+f_{1}^{(2)}+\text { c.c. } \\
& F_{3}=f_{2} z \wedge \hat{\omega}+f_{3} \bar{z} \wedge \hat{\omega}+f_{4} z \wedge j+z \wedge \bar{z} \wedge f_{2}^{(2)}+f_{3}^{(2)} \wedge j+z \wedge f^{(3)}+\text { c.c. } \\
& F_{5}=f_{5} z \wedge j \wedge j+z \wedge \bar{z} \wedge j \wedge f_{4}^{(2)}+\text { c.c. }
\end{aligned}
$$

where $h_{i}$ and $f_{i}$ are complex scalars in the singlet representation of $\mathrm{SU}(2), h_{i}^{(2)}$ and $f_{i}^{(2)}$ are holomorphic vectors in the $\mathbf{2}$ and $h^{(3)}$ and $f^{(3)}$ are complex two forms in the triplet representation, which are $(1,1)$ and primitive with respect to $j$.

Using the above decompositions, the supersymmetry variations can be written as a set of conditions on the torsions classes and the fluxes. The singlets in the torsions must satisfy

$$
\begin{array}{rlrl}
S_{2}=0, & S_{1} & =-S_{4}=-i \mu e^{-A}, \\
S_{3}=\frac{1}{2} \partial_{\bar{z}}(3 A-\phi), & S_{5} & =\bar{S}_{6}=i \bar{h}_{1}-\frac{1}{2} e^{-A} \mu, \\
S_{7} & =\bar{S}_{8}=-\frac{1}{2} \partial_{z}(2 A-\phi),
\end{array}
$$

while the vectors are

$$
\begin{array}{ll}
V_{3}=V_{4}=V_{6}=0, & V_{7}=i\left(\bar{\partial}_{4} A+\bar{h}_{1}^{(2)}\right)\llcorner\omega, \\
V_{5}=i h_{2}^{(2)}, & V_{8}=i\left[\bar{\partial}_{4}(3 A-\phi)+\bar{h}_{1}^{(2)}\right]\llcorner\omega, \\
V_{1}=V_{2}=\partial_{4}(3 A-\phi), &
\end{array}
$$

and the two-forms read

$$
T_{1}=0, \quad T_{2}=-i h^{(3)}, \quad T_{3}=\bar{T}_{4} .
$$

For the fluxes, we find for the NS flux singlets

$$
h_{1}=\bar{h}_{2}, \quad h_{3}=-\frac{i}{2} \partial_{z}(2 A-\phi),
$$

and for the RR fluxes

$$
\begin{aligned}
f_{1} & =e^{-\phi}\left(\frac{1}{2} \bar{\mu} e^{-A}-4 i h_{1}\right), & f_{1}^{(2)} & =i e^{-\phi} \omega\left\llcorner\left[\bar{\partial}_{4}(2 A-\phi)+\bar{h}_{1}^{(2)}\right],\right. \\
f_{2} & =\bar{f}_{3}=-\frac{i}{2} e^{-\phi} \partial_{z} A, & f_{2}^{(2)} & =\frac{i}{2} e^{-\phi} \omega\left\llcorner\left[\bar{\partial}_{4}(4 A-\phi)-\bar{h}_{1}^{(2)}\right],\right. \\
f_{4} & =\frac{1}{2} e^{-\phi}\left(4 h_{1}+i \bar{\mu} e^{-A}\right), & f_{3}^{(2)} & =f_{4}^{(2)}=0, \\
f_{5} & =\frac{3}{4} e^{-A-\phi} \bar{\mu}, & f^{(3)} & =i e^{-\phi} T_{3} .
\end{aligned}
$$

On the fluxes given above we still have to impose the Bianchi identities.

\footnotetext{
${ }^{6} \mathrm{We}$ added two vector representations in $\mathrm{d} z$ that were missing in [41].
} 


\section{A simple class of $\mathrm{SU}(2)$ structure geometries}

A general analysis of the $\mathrm{SU}(2)$ structure constraints derived in the previous section is very involved, due to the large number of torsion classes. In this section we restrict to a subset of all possible $\mathrm{SU}(2)$ structure geometries for which some of the torsion classes are set to zero. Our motivation is to make contact with explicit examples, which are most easily constructed on homogeneous manifolds (groups and cosets) by restricting to left-invariant forms. Moreover we expect to have O-planes in our solutions as they are required to achieve a hierarchy of scales. The possible SU(2) structures one can define out of left-invariant forms, consistent with the orientifold involutions, is restricted. It is this restriction that we consider in this section. Even if we use homogeneous spaces to justify the specific choice of SU(2) torsions, the general solutions we derive could be applicable to more general manifolds.

Following [28], we introduce $\mathrm{O} 5$ and $\mathrm{O} 7$ planes filling the $\mathrm{AdS}_{4}$ directions and mutually intersecting on the internal manifold. Since they are intersecting, we take the O-planes to be smeared on the internal manifold. The orientifold directions are fixed by requiring $\mathcal{N}=1$ supersymmetry ${ }^{7}$ and compatibility with the $\mathrm{SU}(2)$ structure.

The orientifold action on the pure spinors is given by $[16,42]$

$$
\sigma\left(\Phi_{+}\right)= \pm \lambda\left(\bar{\Phi}_{+}\right) \quad \sigma\left(\Phi_{-}\right)=\mp \lambda\left(\Phi_{-}\right)
$$

where $\sigma$ is the orientifold involution, $\lambda$ is the transposition operator (2.6) and the upper and lower signs correspond to $\mathrm{O} 5$ and $\mathrm{O} 7$ planes, respectively. From this we can deduce how the orientifold involution acts on the $\mathrm{SU}(2)$ structure forms. A dynamical $\mathrm{SU}(2)$ structure is not compatible with both $\mathrm{O} 5$ and $\mathrm{O} 7$ projections, since, when $k_{\|} \neq 0$ the phases of the spinors have to be different for $\mathrm{O} 5$ and $\mathrm{O} 7$ planes [42]

$$
\text { O5 }: a= \pm b \quad \text { O7 }: a= \pm i b .
$$

Therefore, all we need is the orientifold action on the rigid $\mathrm{SU}(2)$ structure forms

$$
\begin{aligned}
\sigma(z) & =\mp z, \\
\sigma(j) & =-j, \\
\sigma(\hat{\omega}) & = \pm \overline{\hat{\omega}} .
\end{aligned}
$$

From the equations above we see that the one-form $z$ must be orthogonal to the O5-planes and parallel to the O7's. It is also useful to remind how the NS and RR fluxes transform under the orientifold involutions

$$
\begin{aligned}
\sigma(H) & =-H, \\
\sigma\left(F_{1}\right) & =\mp F_{1}, \\
\sigma\left(F_{3}\right) & = \pm F_{3}, \\
\sigma\left(F_{5}\right) & =\mp F_{5},
\end{aligned}
$$

where, as before, upper and lower signs correspond to O5 and O7-planes.

\footnotetext{
${ }^{7}$ For two O-planes or D-branes to be mutually supersymmetric the number of mixed Neumann and Dirichelet direction must be divisible by four.
} 


\begin{tabular}{|c|c|c|c|c|c|c|}
\hline plane & 1 & 2 & 3 & 4 & 5 & 6 \\
\hline O5 & & & $\mathrm{x}$ & $\mathrm{x}$ & & \\
\hline O5 & & & & & $\mathrm{x}$ & $\mathrm{x}$ \\
\hline O7 & $\mathrm{x}$ & $\mathrm{x}$ & $\mathrm{x}$ & & $\mathrm{x}$ & \\
\hline O7 & $\mathrm{x}$ & $\mathrm{x}$ & & $\mathrm{x}$ & & $\mathrm{x}$ \\
\hline
\end{tabular}

Table 1. O5- and O7-planes.

If we imagine to have a group manifold geometry, we can choose a basis of globally defined one-forms adapted to the product structure defined by the $\mathrm{SU}(2)$ structure (see appendix A) and we identify the directions $e^{1}$ an $e^{2}$ with the real and imaginary part of $z$. Then the most general choice of O-planes is

Notice that these orientifold projections can also be regarded as an asymmetric orbifold of $T^{6} /\left(\mathbb{Z}_{2} \times(-1)^{F_{L}} \mathbb{Z}_{2}\right)$ with one single O-plane. As pointed out in [28], asymmetric orbifolds of this type can be argued to have a valid supergravity description.

The choice of orientifold also constrains the complex structure on $T_{4} M$. One can show that the most general ansatz compatible with the orientifolds of table 1 is

$$
\begin{aligned}
z & =z_{1} e^{1}+z_{2} e^{2}, \\
j & =j_{1} e^{36}+j_{2} e^{45}, \\
\hat{\omega}_{R} & =\frac{j_{1} j_{2}}{\omega_{1}} e^{34}+\omega_{1} e^{56}, \\
\hat{\omega}_{I} & =-\frac{j_{1} j_{2}}{\omega_{2}} e^{35}+\omega_{2} e^{46},
\end{aligned}
$$

where $z_{1}$ and $z_{2}$ are complex number and $j_{1}, j_{2}, \omega_{1}$ and $\omega_{2}$ are real.

The orientifold projections considerably simplify the $\mathrm{SU}(2)$ torsion classes (A.18) and, consequently, the supersymmetry conditions. It is easy to see that all vector representations (doublets) in (2.34) are projected out. The triplet $\tilde{j}_{i}$ of anti self-dual two-forms have the same transformation properties as $j, \omega_{R}$ and $\omega_{I}^{8}$

$$
\sigma\left(\tilde{j}_{1}\right)=-\tilde{j}_{1}, \quad \sigma\left(\tilde{j}_{2}\right)= \pm \tilde{j}_{2} \quad \sigma\left(\tilde{j}_{3}\right)=\mp \tilde{j}_{3} .
$$

As a result the supersymmetry conditions (A.65)-(A.69) reduce to

$$
\begin{aligned}
\mathrm{d} z & =2 \mu e^{-A} \hat{\omega}_{I}, \\
\mathrm{~d} j & =\left(2 i \bar{h}_{1}-\mu e^{-A}\right) \bar{z} \wedge \hat{\omega}_{R}-i z \wedge h^{(3)}+\text { c.c. },
\end{aligned}
$$

${ }^{8}$ The most general choice for the $\tilde{j}_{i}$ compatible with the orientifold projection is

$$
\begin{aligned}
& \tilde{j}_{1}=j_{1} e^{36}-j_{2} e^{45}, \\
& \tilde{j}_{2}=-\frac{j_{1} j_{2}}{\omega_{1}} e^{34}+\omega_{1} e^{56}, \\
& \tilde{j}_{3}=-\frac{j_{1} j_{2}}{\omega_{2}} e^{35}-\omega_{2} e^{46} .
\end{aligned}
$$




$$
\begin{aligned}
\mathrm{d} \hat{\omega}_{R} & =\left(2 i h_{1}+\bar{\mu} e^{-A}\right) z \wedge j+z \wedge T_{3}+\text { c.c. }, \\
\mathrm{d} \hat{\omega}_{I} & =0
\end{aligned}
$$

while the fluxes become

$$
\begin{aligned}
H & =2 h_{1} z \wedge \hat{\omega}_{R}+z \wedge h^{(3)}+\text { c.c. } \\
F_{1} & =e^{-\phi}\left(4 i h_{1}-\frac{1}{2} \bar{\mu} e^{-A}\right) z+\text { c.c. } \\
F_{3} & =-\frac{1}{2} e^{-\phi}\left(i \bar{\mu} e^{-A}+4 h_{1}\right) z \wedge j+\frac{i}{2} e^{-\phi} z \wedge T_{3}+\text { c.c. } \\
F_{5} & =e^{-\phi} f_{5} z \wedge j \wedge j+\text { c.c. }
\end{aligned}
$$

Comparing (3.7) and (3.4) we can see that only one of the three components survive for each $T_{i}$ and $h^{(3)}$

$$
T_{3}=t_{3} \tilde{j}_{1}, \quad h^{(3)}=h_{4} \tilde{j}_{2} .
$$

In all previous equations, since we have smeared O-planes, we assume that all scalars, including the warp factor and the dilaton, are constant.

What remains to be solved are the Bianchi identities (2.20) and (2.22). To do so we need the derivatives of $T_{3}$ and $h^{(3)}$, which can be easily determined from (3.10) and

$$
\begin{aligned}
& \mathrm{d} \tilde{j}_{1}=t_{3} z \wedge \hat{\omega}_{R}-a_{2} z \wedge \tilde{j}_{2}+\text { c.c }, \\
& \mathrm{d} \tilde{j}_{2}=-i h_{4} z \wedge j+a_{2} z \wedge \tilde{j}_{1}+\text { c.c },
\end{aligned}
$$

where the equations above can obtained expanding the $\mathrm{d} \tilde{j}_{i}$ as in (2.34) and imposing the orientifold projections.

Let us start with the BI identities for NS three-form. Using (3.8), (3.9) and (3.11) we obtain

$$
\left|h_{4}\right|^{2}-4\left|h_{1}\right|^{2}+2 \operatorname{Im}\left(e^{-A} \mu h_{1}\right)=0, \quad \operatorname{Im}\left(2 h_{1} \bar{t}_{3}+h_{4} \bar{a}_{2}\right)=0 .
$$

The equation for the five-form flux is trivially satisfied. We are left with the BI involving sources (by abuse of notation we also denote by a $\delta$ the contribution of smeared sources)

$$
\begin{aligned}
& \mathrm{d} F_{1}=\delta(D 7 / O 7) \\
& \mathrm{d} F_{3}=H \wedge F_{1}+\delta(D 5 / O 5) .
\end{aligned}
$$

Using again (3.8) and (3.9), they give

$$
\begin{aligned}
\delta(D 7 / O 7)= & -2 e^{-\phi}\left(\left|e^{-A} \mu\right|^{2}+8 \operatorname{Im}\left(e^{-A} \mu h_{1}\right)\right) \hat{\omega}_{I}, \\
\delta(D 5 / O 5)= & -2 i e^{-\phi}\left(\operatorname{Re}\left(a_{2} \bar{t}_{3}\right)-\operatorname{Im}\left(e^{-A} \mu h_{4}\right)-6 \operatorname{Re}\left(\bar{h}_{1} h_{4}\right)\right) z \wedge \bar{z} \wedge \tilde{j}_{2} \\
& +i e^{-\phi}\left(2\left|t_{3}\right|^{2}+24\left|h_{1}\right|^{2}-\left|e^{-A} \mu\right|^{2}\right) z \wedge \bar{z} \wedge \hat{\omega}_{R} .
\end{aligned}
$$

Notice that the parameters in the previous equations have to satisfy further consistency conditions, namely $\mathrm{d}^{2} j=\mathrm{d}^{2} \hat{\omega}_{R}=0$ and $\mathrm{d}^{2} \tilde{j}_{i}=0$. More precisely, taking the exterior 
derivative of (3.8) and (3.11) we obtain (the consistency conditions on $\tilde{j}_{1}$ and $\tilde{j}_{2}$ give the same equations)

$$
\begin{aligned}
\operatorname{Re}\left(h_{4} \bar{a}_{2}+2 h_{1} \bar{t}_{3}\right)-\operatorname{Im}\left(e^{-A} \mu t_{3}\right) & =0, \\
\operatorname{Re}\left(e^{-A} \mu h_{4}\right)+\operatorname{Im}\left(2 \bar{h}_{1} h_{4}+a_{2} \bar{t}_{3}\right) & =0 .
\end{aligned}
$$

In summary, in order to find a generic $\mathcal{N}=1 \mathrm{AdS}_{4}$ vacua with the choice of O-plane of table 1, one has to solve (3.12), (3.16) and (3.17). The fluxes and the geometry are then given by (3.5) and (3.9). The general solutions to these equations are easy to obtain but, since the expressions are not very illuminating, we do not give them in the paper.

\section{Scale separation}

A question relevant for both compactifications and holography is whether genuine 4dimensional vacua exist within $10 d$ supergravity. To this extent some conditions have to be fulfilled: the string coupling constant $\mathrm{e}^{\phi}$ needs to be tunable small in order to suppress string loop corrections, for $\alpha^{\prime}$ corrections to be small the internal volume needs to be

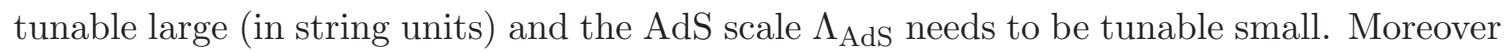
to be able to decouple the massive KK modes and to reduce to a fully 4-dimensional theory, all of these three conditions must combine in such a way that the AdS length scale, $L_{\text {AdS }}$, is parametrically larger than the length scale set by the compact dimensions $L_{\mathrm{KK}}$

$$
\frac{L_{\mathrm{KK}}}{L_{\mathrm{AdS}}} \ll 1 .
$$

Let us first discuss how to define $L_{\mathrm{KK}}$ and $L_{\mathrm{AdS}}$. The four dimensional length scale is set by the inverse of the $\mathrm{AdS}_{4}$ cosmological constant in the four-dimensional Einstein frame. We follow the notation of [43]. Since all solutions we will consider have constant warp factor, we will set $e^{A}=1$. Then we rewrite the 10-dimensional string frame metric (2.5) as

$$
\mathrm{d} s_{10}^{2}=\tau_{0}^{2} \tau^{-2} \mathrm{~d} s_{4}^{2}+\rho \mathrm{d} \tilde{s}_{6}^{2},
$$

where $\mathrm{d} s_{4}^{2}$ is the 4 -dimensional Einstein frame metric. We have rescaled the internal metric

$$
\mathrm{d} s_{6}^{2}=\rho \mathrm{d} \tilde{s}_{6}^{2},
$$

in such a way that the modulus $\rho=\left(\operatorname{det} g_{6}\right)^{1 / 6}$ measures the string frame volume of the internal manifold and

$$
\int_{6} \sqrt{\tilde{g}_{6}}=\mathcal{O}(1) .
$$

The variable $\tau$ is the 4-dimensional dilaton and is given by

$$
\tau^{2}=\mathrm{e}^{-2 \phi} \rho^{3} .
$$

With $\tau_{0}$ we denote the VEV of $\tau$, such that in equation (4.2) only the dynamical part of $\tau$ is used to obtain $4 d$ Einstein frame. 
Then direct dimensional reduction of the 10-dimensional string frame action gives the $4 d$ Planck mass in terms of the string mass scale $M_{s}^{2}$

$$
m_{p}^{2}=\tau_{0}^{2} M_{s}^{2}
$$

We define the dimensionally reduced action as

$$
S=\int \sqrt{g}\left(m_{p}^{2} R-V\right)
$$

such that the scalar potential is a dimension four operator. The AdS cosmological constant is then defined as

$$
\Lambda_{\mathrm{AdS}}=\frac{V}{m_{p}^{2}} .
$$

The number $|\mu|^{2}$ that appears in the supersymmetry equations of the previous section is related to the cosmological constant in the following way

$$
|\mu|^{2}=-6 \Lambda_{\mathrm{AdS}}
$$

We define the AdS length scale as $L_{\text {AdS }}^{2}=-\Lambda_{\text {AdS }}^{-1}$, such that it determines the $4 d$ curvature as follows

$$
2 R_{\mu \nu}^{(4)}=L_{\mathrm{AdS}}^{-2} g_{\mu \nu}^{(4)} .
$$

The size of the internal manifold is less straightforward to define. A natural guess for the KK scale, which we will adopt in this paper, is

$$
L_{\mathrm{KK}}^{2}=\rho .
$$

The proper way to check the condition (4.1) would be to compute the Kaluza-Klein spectrum and see that the masses are indeed much larger than the AdS scale. Since this is often not easy to perform, one has to rely on some simpler estimates this ratio.

A way to determine under which conditions scale separation can be achieved is to study the dependence of the effective four-dimensional potential on two moduli, namely the volume of the compact manifold, $\rho$, and the dilaton, $\phi$. As an example we briefly recall how the this can be applied to the model of [6], which is one of the first constructions of a type IIA vacuum admitting full moduli stabilisation and scale separation. The model of [6] corresponds to a compactification on an orbifold of $T^{6}$ with non-zero $F_{0}, F_{4}, H$-fluxes and O6 sources. The scalar potential depends on the moduli $\tau$ and $\rho$ schematically as

$$
V(\rho, \tau) / m_{p}^{4}=|H|^{2} \rho^{-3} \tau^{-2}+T_{\mathrm{O} 6} \tau^{-3}+\left|F_{0}\right|^{2} \tau^{-4} \rho^{3}+\left|F_{4}\right|^{2} \tau^{-4} \rho^{-1},
$$

where $T_{\mathrm{O} 6}$ is the $\mathrm{O} 6$ tension and is the only negative term in the potential. The coefficients in the potential are a priori functions of the other moduli. In the particular example of [6], it can be shown that, while the $H$ and $F_{0}$ flux are constrained by the tadpole condition to be order one, the $F_{4}$ flux is an unbounded flux quantum. In what follows we assume that $|H|^{2}, T_{O 6},\left|F_{0}\right|^{2}$ are all order one in proper units and $\left|F_{4}\right|^{2}$ scales as $N^{2}$, where $N$ is 
unbounded. From the detailed balance condition (all terms are of the same order in the potential) we can derive the $N$-dependence for $\rho$ and $\tau$ at a critical point

$$
\rho \sim N^{\frac{1}{2}}, \quad \tau \sim N^{\frac{3}{2}} \quad\left(\mathrm{e}^{\phi} \sim N^{-\frac{3}{4}}\right)
$$

from which we see that large $N$ implies large volume and weak coupling. Secondly, we find that the AdS scale becomes tunably small in the same limit. Using the scaling of the potential and the $4 d$ Planck mass we find

$$
V / m_{p}^{4} \sim N^{-\frac{9}{2}} \quad m_{p}^{2} \sim M_{s}^{2} \mathrm{e}^{-2 \phi} \rho^{3} \sim N^{3} \quad L_{\mathrm{AdS}}^{-2} \sim N^{-3 / 2} .
$$

Since $\rho=L_{\mathrm{KK}}^{2}$ we indeed find scale separation

$$
\frac{L_{\mathrm{KK}}}{L_{\mathrm{AdS}}} \rightarrow 0
$$

A similar argument can be also given for IIB solutions. On the type IIB side, an explicit example with the right properties of tunably large volume, small coupling and small AdS scale was found in [8] by T-dualising the type IIA torus example. A systematic study, from a $4 d$ point of view, of IIB solutions was initiated in [28]. Typically we have models with $F_{1}, F_{3}, F_{5}$ flux, O5, O7 sources on some curved internal manifold. The scalar potential can be written as

$$
V(\rho, \tau) / m_{p}^{4}=\tau^{-4}\left(\left|F_{5}\right|^{2} \rho^{-2}+\left|F_{3}\right|^{2}+\left|F_{1}\right|^{2} \rho^{2}\right)+\tau^{-3}\left(T_{O 7} \rho^{\frac{1}{2}}+T_{O 5} \rho^{-\frac{1}{2}}\right)+R_{6} \tau^{-2} \rho^{-1} .
$$

where, as in the type IIA case, the coefficients are functions of all other moduli. The models of $[8,28]$ are characterised by two unbounded flux quanta: $F_{5}$ and a component of $F_{1}$, whereas another component is determined by the $\mathrm{O} 7$ planes and not tunable. So let us scale both fluxes

$$
\left|F_{5}\right|^{2} \sim N^{2} \quad\left|F_{1}\right|^{2} \sim N^{C}
$$

where $C$ is some positive number. If we then balance $F_{5}^{2}$ against $F_{1}^{2}$ and $T_{\mathrm{O} 7}$ we find the following $N$-dependence

$$
\rho \sim N^{\frac{1}{2}-\frac{C}{4}}, \quad \mathrm{e}^{\phi} \sim N^{-\frac{C}{4}} .
$$

If $0<C<2$ the solution is indeed at large volume and weak coupling for large $N$. The $F_{5}, F_{1}, T_{\mathrm{O} 7}$ contributions, which set the size of the AdS solution, scale as

$$
V / m_{p}^{4} \sim N^{-2-2 C}
$$

and go to zero at large $N$. If we compute $L_{\text {AdS }}^{2}$ we again find a separation of scales. This argument relied on a detailed balance condition for the $F_{5}, F_{1}$ and $T_{\mathrm{O} 7}$ contributions. We have not discussed the other contributions to the scalar potential. In the explicit solutions we derive in this paper all terms in the potential will be of the same order of magnitude.

The Ricci scalar of the internal space scales as the inverse metric/ $\rho^{-1}$. Therefore one would be tempted to conclude that in the large volume limit the two definitions of scale 
separation

$$
\begin{array}{ll}
\text { scale separation (1) : } & \frac{L_{\mathrm{AdS}}^{2}}{L_{\mathrm{KK}}^{2}} \rightarrow \infty, \\
\text { scale separation (2) : } & \frac{R_{6}}{R_{4}} \rightarrow \infty,
\end{array}
$$

are equivalent. However, these two definitions do not need to coincide if the normalised curvature $\tilde{R}$

$$
R_{6}=\rho^{-1} \tilde{R}\left(\phi_{I}\right),
$$

is not kept constant when the limit of large $\rho$ is taken. The other moduli $\phi_{I}$ that appear inside the normalised curvature could also introduce an extra scaling. Below we find an explicit examples for which there is scale separation according to the first definition, but not according to the second definition. Nonetheless notice that, as we will show in the next sections, the ratio of the Ricci scalars can be very useful in setting general conditions on the torsion classes of the internal manifold in order to achieve separation of scales.

\subsection{Separation of scales without sources?}

The most trustworthy solutions are those without any orientifold or D-brane sources since there is no reason to worry about the smearing approximation or charge quantisation. Even in the case one knows the localised solutions one could have rightful worries about the use of supergravity in the presence of singular sources. A priori, sourceless AdS SUSY vacua can exist both for SU(3) structure manifolds IIA and SU(2) structure ones in IIB. When SUSY is broken many more solutions can exist, see for instance reference [10] for the IIA case. It is not clear whether solutions without sources allow for separation of scales [11]. The above scaling arguments do not obviously use the presence of a source term. We did include it in the analysis, but we could have equally discarded it. It turns out that it depends on the details of the manifold whether there exists the specific large flux limits that achieve scale separation. While no no-go theorem has been found so far, there is no example known of a solution in 10d SUGRA, whether SUSY or not, that achieves scale separation without sources.

In the following we give a simple argument that seems to suggest that AdS without sources do not allow for scale separation. The argument below holds under two assumptions: 1) there is no warping and 2) the size of the internal manifold cannot be decoupled from its curvature radius.

Consider a general compactification with $\mathrm{RR}$ fluxes $F_{p}, H$ flux and no sources. The scalar potential can be written as

$$
V(\rho, \tau) / m_{p}^{4}=-R_{6} \tau^{-2} \rho^{-1}+|H|^{2} \rho^{-3} \tau^{-2}+\sum_{p}\left|F_{p}\right|^{2} \rho^{3-p} \tau^{-4} .
$$

The vacua of the theory must be extrema of the scalar potential. One can easily verify that the equations

$$
\partial_{\rho} V=0, \quad \partial_{\tau} V=0
$$

are specific linear combinations of the dilaton equation of motion in 10 dimensions, the trace over the internal indices of the (trace reversed) Einstein equation, and the external 
Einstein equation [44]. Upon eliminating $|H|^{2}$ in terms of the RR field strength densities, these are equivalent to

$$
\begin{aligned}
& R_{4}=2 V=-2 \sum_{p}\left|F_{p}\right|^{2}<0, \\
& R_{6}=\sum_{p} \frac{9-p}{2}\left|F_{p}\right|^{2}>0,
\end{aligned}
$$

where we did not write down the explicit $\rho, \tau$ dependence anymore. The first condition is an alternative derivation of the Maldacena-Nunez no-go theorem [45] in the simple case of no warping. The second condition was found before [46]. With the above equations we can compute the ratio

$$
r=\left|\frac{R_{6}}{R_{4}}\right|,
$$

and define scale separation as the possibility to have $r \gg 1$ (4.21). However, our equations imply that $r$ is bounded from above by a number $r_{\max }$, since $p<9$. We compute $r_{\max }$ by rewriting the inequality:

$$
\sum_{p}\left(\frac{9-p}{2}-2 r_{\max }\right)\left|F_{p}\right|^{2}<0 .
$$

From this one deduces that

$$
r_{\max }=\frac{9-p_{\max }}{4}
$$

where $p_{\max }$ is the highest rank field strength that is turned on in the vacuum solution. We then conclude that, under the assumptions we discussed above, AdS vacua not supported by sources cannot achieve scale separation. Note that indeed Freund-Rubin vacua [47] have $r$ of order one. It would be most interesting to see whether the same argument holds when allowing for warping.

\section{$5 \quad$ Explicit examples}

Natural candidates for our search for vacua are manifolds for which we can compute the $\mathrm{SU}(2)$ structure explicitly. These are typically coset and group manifolds, although recently progress has been made for non-homogeneous manifolds [48, 49]. In this paper we study in details the class of coset manifolds discussed in [28] and nilmanifolds. Our aim is to compute the $10 d$ solutions when they exist and to demonstrate when solutions cannot exist. This was an open issue in [28] and with the pure spinor technology this is quite straightforward to settle.

Group manifolds are particularly simple to analyse because of the existence of leftinvariant forms, which can be used as an expansion basis for the various fields on the manifold. In particular, one can also take the forms defining the $\mathrm{SU}(2)$ structure to be left-invariant. Let us recall, for simplicity, that the most general ansatz for the SU(2) structure forms and the anti-self dual triplet $\tilde{j}_{1}, \tilde{j}_{2}$ and $\tilde{j}_{3}$ compatible with the orientifold projections of table 1 is

$$
\begin{aligned}
& z=z_{1} e^{1}+z_{2} e^{2}, \\
& j=j_{1} e^{36}+j_{2} e^{45}
\end{aligned}
$$




$$
\begin{aligned}
& \omega_{R}=\frac{j_{1} j_{2}}{\omega_{1}} e^{34}+\omega_{1} e^{56}, \\
& \omega_{I}=-\frac{j_{1} j_{2}}{\omega_{2}} e^{35}+\omega_{2} e^{46},
\end{aligned}
$$

with $z_{1}=z_{1 R}+i z_{1 I}, z_{2}=z_{2 R}+i z_{2 I}$ and

$$
\begin{aligned}
& \tilde{j}_{1}=j_{1} e^{36}-j_{2} e^{45}, \\
& \tilde{j}_{2}=-\frac{j_{1} j_{2}}{\omega_{1}} e^{34}+\omega_{1} e^{56}, \\
& \tilde{j}_{3}=-\frac{j_{1} j_{2}}{\omega_{2}} e^{35}-\omega_{2} e^{46} .
\end{aligned}
$$

The NS and RR fluxes are given by (3.9)

$$
\begin{aligned}
H & =2 h_{1} z \wedge \hat{\omega}_{R}+h_{4} z \wedge \tilde{j}_{2}+\text { c.c. } \\
F_{1} & =e^{-\phi}\left(4 i h_{1}-\frac{1}{2} \bar{\mu} e^{-A}\right) z+\text { c.c. } \\
F_{3} & =-\frac{1}{2} e^{-\phi}\left(i \bar{\mu} e^{-A}+4 h_{1}\right) z \wedge j+\frac{i}{2} e^{-\phi} t_{3} z \wedge \tilde{j}_{1}+\text { c.c. } \\
F_{5} & =e^{-\phi} f_{5} z \wedge j \wedge j+\text { c.c. }
\end{aligned}
$$

For left-invariant forms, all the exterior derivatives are given in terms of the structure constants and the supersymmetry constraints (3.8) reduce to algebraic equations. The computation then proceeds by enforcing all the algebraic conditions, those coming from supersymmetry, the Bianchi identities (3.12) and the consistency conditions (3.16), (3.17). Finally (3.15) is used to determine the source terms.

We can use (2.12) to derive the volume of the internal manifold

$$
\operatorname{vol}_{6}=\left.\frac{i}{4} z \wedge \bar{z} \wedge j \wedge j\right|_{\text {top }}=j_{1} j_{2} \operatorname{Im}\left(z_{1} \bar{z}_{2}\right),
$$

and its metric (see [16])

$$
g_{i j}=\left(\begin{array}{cccccc}
z_{r 1}^{2}+z_{i 1}^{2} & z_{r 1} z_{r 2}+z_{i 1} z_{i 2} & 0 & 0 & 0 & 0 \\
z_{r 1} z_{r 2}+z_{i 1} z_{i 2} & z_{r 2}^{2}+z_{i 2}^{2} & 0 & 0 & 0 & 0 \\
0 & 0 & -\frac{j_{1}^{2} j_{2}}{\omega_{1} \omega_{2}} & 0 & 0 & 0 \\
0 & 0 & 0 & -\frac{j_{2} \omega_{2}}{\omega_{1}} & 0 & 0 \\
0 & 0 & 0 & 0 & -\frac{j_{2} \omega_{1}}{\omega_{2}} & 0 \\
0 & 0 & 0 & 0 & 0 & -\frac{\omega_{1} \omega_{2}}{j_{2}}
\end{array}\right) .
$$

For this class of models, the Ricci scalar takes a very simple form when expressed in terms of the $\mathrm{SU}(2)$ torsion classes

$$
R_{6}=-4\left(\left|S_{1}\right|^{2}+4\left|S_{5}\right|^{2}+\operatorname{Im}\left(S_{5} \bar{S}_{1}\right)+\left|T_{2}\right|^{2}+\left|T_{3}\right|^{2}\right) .
$$


We are also interested in the total charges associated with the sources. These can be obtained form (3.15) writing the Bianchi identities (2.22) $\mathrm{as}^{9}$

$$
\begin{aligned}
\mathrm{d} F_{1} & =\sum_{i} N_{O 7 / D 7}^{i} \eta_{2}^{i} \\
\mathrm{~d} F_{3}-H \wedge F_{1} & =\sum_{i} N_{O 5 / D 5}^{i} \eta_{4}^{i},
\end{aligned}
$$

where $\eta_{2}^{i}$ and $\eta_{4}^{i}$ are decomposable two- and four-forms Poincaré dual to the cycles wrapped by the sources.

The numbers, $N_{O 5 / D 5}^{(i)}$ and $N_{O 7 / D 7}^{(i)}$ are related to the total charges and these should typically be of order one on a solution since they are directly related to the number of Dbranes or orientifolds. From the Bianchi identity one can not say whether the sources correspond to orientifolds, D-branes, or a certain mixture. This can be read of from the tension terms in the Einstein equation, combined with charge quantisation. Determining whether D-branes are present is clearly relevant for moduli stabilisation, since they introduce extra open string moduli, but it is beyond the scope of this paper. It is worth mentioning that in IIA it is possible to have solutions where only O-planes are present: this is is the case, for instance, of the prime example of [6] where the sources are purely smeared O6 planes.

Deriving (5.3), it is straightforward to check that the O7/D7-plane charges are

$$
\begin{aligned}
& N_{O 7 / D 7}^{(1)}=2 e^{-\phi} \frac{j_{1} j_{2}}{\omega_{2}}\left[|\mu|^{2}+8 \operatorname{Im}\left(\mu h_{1}\right)\right], \\
& N_{O 7 / D 7}^{(2)}=-2 e^{-\phi} \omega_{2}\left[|\mu|^{2}+8 \operatorname{Im}\left(\mu h_{1}\right)\right],
\end{aligned}
$$

while for the O5/D5 we obtain

$$
\begin{aligned}
& N_{O 5 / D 5}^{(1)}=2 e^{-\phi} \frac{j_{1} j_{2}}{\omega_{1}} \operatorname{Im}\left(z_{1} \bar{z}_{2}\right)\left[2\left|t_{3}\right|^{2}-|\mu|^{2}+24\left|h_{1}\right|^{2}-2 \operatorname{Im}\left(\mu h_{4}\right)-2 \operatorname{Re}\left(6 h_{4} \bar{h}_{1}-a_{2} \bar{t}_{3}\right)\right], \\
& N_{O 5 / D 5}^{(2)}=2 e^{-\phi} \omega_{1} \operatorname{Im}\left(z_{1} \bar{z}_{2}\right)\left[2\left|t_{3}\right|^{2}-|\mu|^{2}+24\left|h_{1}\right|^{2}+2 \operatorname{Im}\left(\mu h_{4}\right)+2 \operatorname{Re}\left(6 h_{4} \bar{h}_{1}-a_{2} \bar{t}_{3}\right)\right] .
\end{aligned}
$$

Before moving to the discussion of the explicit examples we summarise in table 2 whether our solutions admit weak coupling, large volume and scale separation. More precisely we look at possible scalings where, taking the limit of small cosmological constant

$$
|\mu|^{2} \rightarrow 0
$$

we can have small coupling and large volume. As can be seen from table 2 we have also checked whether separation of scales according to the two definitions (4.20), (4.21) is possible. Only the solutions on Nil 4.1 and 5.1 can be tuned into a trustworthy regime. The solution on Nil 3.14 cannot be achieved for large volume and furthermore suffers from having a singular limit (vanishing volume) if scale separation is required. We also notice that for several examples there is no match between the two criteria for scale separation.

In finding the appropriate limits we have taken a conservative and safe viewpoint where each source term was taken to have finite prefactors as a consequence of charge quantisation

\footnotetext{
${ }^{9} \mathrm{~A}$ derivation of this expression can be found in appendix $\mathrm{C}$.
} 


\begin{tabular}{|c|c|c|c|c|}
\hline Manifold & weak coupling & Large volume & scale separation (1) & scale separation (2) \\
\hline$\frac{\mathrm{SU}(3)}{\mathrm{SU}(2)} \times \mathrm{U}(1)$ & $\times$ & $\checkmark$ & $\checkmark$ & $\times$ \\
\hline$\frac{\mathrm{SU}(2)^{2}}{\mathrm{U}(1)} \times \mathrm{U}(1)$ & $\times$ & $\checkmark$ & $\checkmark$ & $\times$ \\
\hline Nil 3.14 & $\checkmark$ & $\times$ & $\checkmark$ & $\checkmark$ \\
\hline Nil 4.1 & $\checkmark$ & $\checkmark$ & $\checkmark$ & $\checkmark$ \\
\hline Nil 5.1 & $\checkmark$ & $\checkmark$ & $\checkmark$ & $\times$ \\
\hline
\end{tabular}

Table 2. The scaling regimes for the various manifolds with SUSY AdS vacua.

(see footnote below equation (2.22)). Since not all source terms are represented by forms in the cohomology of the internal space, it is possible that charge quantisation is less restrictive on such forms and that certain numbers do not need to take fixed values. In that case it is possible that certain solutions do allow weak coupling and scale separation although the above table indicates otherwise. We leave such subtle issues for further investigation.

\subsection{Coset manifolds}

We refer to [33] for a thorough discussion of coset manifolds and $G$-structures. Here we simply recall some simple facts that help making our derivation clearer.

A coset manifold $M=G / H$ where $G$ is a Lie group and $H$ is a closed subgroup of $G$, is completely determined by the corresponding algebrae, $g$ and $h$. We denote by $\left\{H_{a}\right\}$, with $a=1, \ldots \operatorname{dim} H$, a basis of generators of $h$ and by $\left\{K_{i}\right\}$, with $i=1, \ldots, \operatorname{dim} G-\operatorname{dim} H$ a basis for the complement of $h$ in $g$. Then the structure constants are given by

$$
\begin{aligned}
& {\left[H_{a}, H_{b}\right]=f_{a b}^{c} H_{c},} \\
& {\left[H_{a}, K_{i}\right]=f_{a i}^{j} K_{j}+f_{a i}^{c} H_{c},} \\
& {\left[K_{i}, K_{j}\right]=f_{j k}^{i} K_{i}+f_{j k}^{a} H_{a} .}
\end{aligned}
$$

The coframe $e^{i}(y)$ on $G / H$ is defined by

$$
L^{-1} \mathrm{~d} L=e^{i} K_{i}+\omega^{a} H_{a},
$$

where $L(y)$ is a coset representative and $y^{i}$ are local coordinates on $G / H$. A $p$-form

$$
\phi=\phi_{i_{i} \ldots i_{p}} e^{i_{1}} \wedge \ldots \wedge e^{i_{p}}
$$

is then said to be left-invariant under the action of $G$ if and only if its coefficients $\phi_{i_{i} \ldots i_{p}}$ are constant and

$$
f_{a\left[i_{1}\right.}^{j} \phi_{\left.i_{2} \ldots i_{p}\right] j}=0
$$

From the algebra (5.11) we have

$$
\mathrm{d} e^{i}=-\frac{1}{2} f_{j k}^{i} e^{j} \wedge e^{k}-f_{a j}^{i} \omega^{a} \wedge e^{j} .
$$

It is then easy to show that (5.14) guarantees that the exterior derivative preserve the property of left-invariance. 
As mentioned before, we want the $\mathrm{SU}(2)$ structure to be also left-invariant. As shown in [33] this requires that $H \in \mathrm{SU}(2)$. The list of reductive coset manifold that satisfy this property is [28]

$$
\frac{\mathrm{SU}(3) \times \mathrm{U}(1)}{\mathrm{SU}(2)} \quad \frac{\mathrm{SU}(2)^{2}}{\mathrm{U}(1)} \times \mathrm{U}(1) \quad \mathrm{SU}(2) \times \mathrm{SU}(2) \quad \mathrm{SU}(2) \times \mathrm{U}(1)^{3} .
$$

The rest of this section is devoted to the study of $\mathcal{N}=1$ SUSY $\mathrm{AdS}_{4}$ on such manifolds.

\subsection{1 $\frac{\mathrm{SU}(3) \times \mathrm{U}(1)}{\mathrm{SU}(2)}$}

Of the 9 generators of $\mathrm{SU}(3) \times \mathrm{U}(1)$ we denote by $T_{2}$ and $T_{7}, T_{8}, T_{9}$ the generators of $\mathrm{U}(1)$ and $\mathrm{SU}(2)$, respectively. The algebra is given by

$$
\begin{aligned}
& f_{46}^{1}=-\frac{\sqrt{3}}{2} \quad(\text { and cyclic }), \quad f_{35}^{1}=\frac{\sqrt{3}}{2} \quad(\text { and cyclic }), \\
& f_{78}^{9}=1 \quad(\text { and cyclic }), \\
& f_{65}^{7}=f_{34}^{7}=f_{63}^{8}=f_{45}^{8}=f_{64}^{9}=f_{53}^{9}=\frac{1}{2} \quad(\text { and cyclic }) .
\end{aligned}
$$

The left-invariant forms compatible with the $\mathrm{O} 5$ and $\mathrm{O} 7$ projections are

$$
\begin{array}{ll}
1 \text { forms } & e^{1}, e^{2}, \\
2 \text { forms } & e^{36}+e^{45}, e^{34}+e^{56}, e^{35}-e^{46},
\end{array}
$$

Since the SU(2) structure forms must also be left-invariant, in the ansatz (5.1) and (5.2) we set

$$
j_{1}=j_{2} \quad \omega_{1}=\epsilon_{1} j_{2} \quad \omega_{2}=\epsilon_{2} j_{2},
$$

with $\epsilon_{1}= \pm 1$ and $\epsilon_{2}= \pm 1$. It is easy to see that none of the forms $\tilde{j}_{i}$ is left-invariant, which implies $t_{3}=h_{4}=0$. Solving the constraints (3.12), (3.16) and (3.17) gives the solution

$$
\begin{aligned}
h_{1} & =\frac{i}{2} \frac{\operatorname{Re}\left(\mu \bar{z}_{2}\right)}{z_{2}}, \\
z_{1} & =-\frac{\epsilon_{1}}{2} \frac{\sqrt{3} \mu\left|z_{2}\right|^{2}}{\operatorname{Im}\left(\mu \bar{z}_{2}\right)^{2}}, \\
j_{2} & =-\epsilon_{1} \epsilon_{2} \frac{3}{8} \frac{\left|z_{2}\right|^{2}}{\operatorname{Im}\left(\mu \bar{z}_{2}\right)^{2}}, \\
a_{2} & =0,
\end{aligned}
$$

where

$$
\begin{aligned}
\rho^{3} & =-\epsilon_{1} \frac{9 \sqrt{3}}{128} \frac{\left|z_{2}\right|^{6}}{\operatorname{Im}\left(\mu \bar{z}_{2}\right)^{5}}, \\
R_{6} & =-4\left[|\mu|^{2}-2 \frac{\operatorname{Im}\left(\mu \bar{z}_{2}\right)^{2}}{\left|z_{2}\right|^{2}}\right],
\end{aligned}
$$


and the orientifold charges are

$$
\begin{aligned}
& N_{O 7 / D 7}^{(1)}=N_{O 7 / D 7}^{(2)}=-\frac{3 \epsilon_{1}}{4} e^{-\phi}\left[1+5 \frac{\operatorname{Re}\left(\mu \bar{z}_{2}\right)^{2}}{\operatorname{Im}\left(\mu \bar{z}_{2}\right)^{2}}\right], \\
& N_{O 5 / D 5}^{(1)}=N_{O 5 / D 5}^{(2)}=\frac{3 \sqrt{3}}{8} \epsilon_{1} \epsilon_{2} e^{-\phi}\left|z_{2}\right|^{2}\left[-\frac{1}{\operatorname{Im}\left(\mu \bar{z}_{2}\right)}+5 \frac{\operatorname{Re}\left(\mu \bar{z}_{2}\right)^{2}}{\operatorname{Im}\left(\mu \bar{z}_{2}\right)^{3}}\right] .
\end{aligned}
$$

Note that by consistency the orientifold planes should wrap directions whose dual source forms should be left-invariant. The source forms that are Poincare dual to the surfaces wrapped by the $\mathrm{O} 5$ planes are not left-invariant, although we have said O planes have to be consistent with the left-invariant forms. This problem is however cured since the sum of the two O5 forms, $e^{1234}+e^{1256}$ is left-invariant. If we interpret each of these two terms as a separate orientifold source with its own involution then consistency requires the two Oplanes to have exactly the same charge, such that the source term in the Bianchi identity for $F_{3}$ is given by the sum of the two forms, and hence left-invariant. This is anyhow a necessary requirement from the point of view of charge quantisation. Orientifolds, unlike D-branes, cannot be stacked. So for each involution we should have a single unit of orientifold charge.

\section{$5.1 .2 \frac{\mathrm{SU}(2)^{2}}{\mathrm{U}(1)} \times \mathrm{U}(1)$}

For this coset, out of the 7 generators of $\mathrm{SU}(2)^{2} \times \mathrm{U}(1)$ we denote by $T_{7}$ the generator of the $\mathrm{U}(1)$. The algebra is given by:

$$
\begin{array}{ll}
f_{35}^{1}=1(\text { and cyclic }), & f_{46}^{7}=1(\text { and cyclic }) \\
f_{46}^{1}=f_{37}^{5}=-f_{57}^{3}=-1 . &
\end{array}
$$

As in the previous case, the $\mathrm{SU}(2)$ structure must be left-invariant. This means that in the ansatz (5.1) and (5.2) we set

$$
j_{1}=j_{2} \quad \omega_{1}=\epsilon_{1} j_{2},
$$

with $\epsilon_{1}= \pm 1$. As before, the requirement of left-invariant implies $h_{4}=0$ and $t_{3}=0$. The solution is

$$
\begin{aligned}
& z_{1}=-\epsilon_{1} \frac{\mu\left|z_{2}\right|^{2}}{2 \operatorname{Im}\left(\mu \bar{z}_{2}\right)^{2}}, \\
& \omega_{2}=-\epsilon_{1} \frac{\left|z_{2}\right|^{2}}{4 \operatorname{Im}\left(\mu \bar{z}_{2}\right)^{2}}, \\
& j_{2}=-\epsilon_{2} \frac{\left|z_{2}\right|^{2}}{4 \operatorname{Im}\left(\mu \bar{z}_{2}\right)^{2}}, \\
& h_{1}=\frac{i}{2} \frac{\operatorname{Re}\left(\mu \overline{z_{2}}\right)}{z_{2}}, \\
& h_{4}=t_{3}=0, \\
& a_{2}=i \frac{\operatorname{Im}\left(\mu \bar{z}_{2}\right)}{z_{2}},
\end{aligned}
$$


with $\epsilon_{2}= \pm 1$. The volume and curvature are

$$
\begin{aligned}
\rho^{3} & =\frac{\epsilon_{1}}{32} \frac{\left|z_{2}\right|^{6}}{\operatorname{Im}\left(\mu \bar{z}_{2}\right)^{5}}, \\
R_{6} & =-4\left[|\mu|^{2}-2 \frac{\operatorname{Im}\left(\mu \bar{z}_{2}\right)^{2}}{\left|z_{2}\right|^{2}}\right],
\end{aligned}
$$

and the orientifold charges are

$$
\begin{aligned}
& N_{O 7}^{(1)}=N_{O 7}^{(2)}=-\frac{\epsilon_{1}}{2} e^{-\phi}\left[1+5 \frac{\operatorname{Re}\left(\mu \bar{z}_{2}\right)}{\operatorname{Im}\left(\mu \bar{z}_{2}\right)}\right], \\
& N_{O 5}^{(1)}=N_{O 5}^{(2)}=\frac{\epsilon_{2}}{4} e^{-\phi}\left|z_{2}\right|^{2}\left[-\frac{1}{\operatorname{Im}\left(\mu \bar{z}_{2}\right)}+5 \frac{\operatorname{Re}\left(\mu \bar{z}_{2}\right)^{2}}{\operatorname{Im}\left(\mu \bar{z}_{2}\right)^{3}}\right], \\
& n_{O 7}^{(1)}=n_{O 7}^{(2)}=-\frac{e^{-\phi}}{4}\left[|\mu|^{2}+4 \frac{\operatorname{Re}\left(\mu \bar{z}_{2}\right)^{2}}{\left|z_{2}\right|^{2}}\right], \\
& n_{O 5}^{(1)}=n_{O 5}^{(2)}=\frac{e^{-\phi}}{4}\left[|\mu|^{2}-6 \frac{\operatorname{Re}\left(\mu \bar{z}_{2}\right)^{2}}{\left|z_{2}\right|^{2}}\right] .
\end{aligned}
$$

\subsection{3 $\quad \mathrm{SU}(2) \times \mathrm{SU}(2)$ and $\mathrm{SU}(2) \times \mathrm{U}(1)^{3}$}

There are no SUSY solutions on these two manifolds. This is most easily seen for $\mathrm{SU}(2) \times$ $\mathrm{SU}(2)$ since the SUSY equations (see (3.8)) require the one-form $\operatorname{Im}(\bar{\mu} z)$ to be closed whereas there are no closed (left-invariant) one-forms on $\mathrm{SU}(2) \times \mathrm{SU}(2)$.

\subsection{Nilmanifolds}

Nilmanifolds have Lie groups defined by a nilpotent algebra as their covering space. Having negative curvature they are natural candidates for flux compactifications [16]. In six dimensions there are 34 isomorphism classes of simply-connected six-dimensional nilpotent Lie groups. The full list of algebrae can be found in table 4 of [16] and in [50], together with the pure spinors and orientifold projections compatible with each algebra. In particular it is easy to see that very few algebrae are compatible with the orientifold projections we are imposing. Using the notation of $[16]^{10}$ they are

Most of the algebrae above are ruled out by the first SUSY condition in (3.8)

$$
\mathrm{d} z=2 \mu \hat{\omega}_{I}
$$

Indeed using the definition of the $\mathrm{SU}(2)$ structure (5.1), it easy to see that only the algebrae $n 3.13, n 3.14, n 4.1$ and $n 5.1$ have structure constants suitable to satisfy this condition. In the rest of this section we give the $\mathcal{N}=1 \mathrm{AdS}_{4}$ solutions for the manifolds $n 3.14, n 4.1$ and $n 5.1$ ( $n 3.13$ is almost identical to $n 3.14$ ). Notice, that differently from the solutions on coset manifolds, these vacua can have non trivial torsion $t_{3}$ and H-flux $h_{4}$.

On a nilmanifold all forms are left-invariant. To our purposes this means that the solutions are less constrained then for coset manifolds. In particular we have no constraints on the moduli in the SU(2) structure. For this reason we expect this vacua to have generically some unfixed moduli.

\footnotetext{
${ }^{10}$ With respect to table 4 of [16] we have relabeled the one-forms in order to match our orientifold projections.
} 


\begin{tabular}{|l|l|c|c|}
\hline$n$ & Nilmanifold class & $b_{1}$ & $b_{2}$ \\
\hline 3.3 & $(0,35,0,0,13,14)$ & 3 & 6 \\
\hline 3.13 & $(-35-46,0,0,0,23,-24)$ & 3 & 5 \\
\hline 3.14 & $(-35+46,0,0,0,23,-24)$ & 3 & 5 \\
\hline 4.1 & $(-35-46,0,-25,0,0,0)$ & 4 & 6 \\
\hline 4.2 & $(-35,0,-25,0,0,0)$ & 4 & 7 \\
\hline 4.5 & $(-46,0,25,0,0,0)$ & 4 & 8 \\
\hline 4.6 & $(0,0,0,0,13,14)$ & 4 & 9 \\
\hline 5.1 & $(35+46,0,0,0,0,0)$ & 5 & 9 \\
\hline 5.2 & $(35,0,0,0,0,0)$ & 5 & 11 \\
\hline 6.1 & $(0,0,0,0,0,0)$ & 6 & 15 \\
\hline
\end{tabular}

Table 3. Six-dimensional nilmanifolds compatible with the O5/O7 projections.

\subsubsection{Model 3.14}

The solution is given by

$$
\begin{aligned}
z_{1} & =-2 \mu \epsilon_{1} \sqrt{j_{1} j_{2}}, \\
\omega_{2} & =\epsilon_{1} \sqrt{j_{1} j_{2}}, \\
h_{1} & =\frac{i}{4} \bar{\mu}\left(2+\frac{i\left(j_{1}+j_{2}\right) \omega_{1}}{2 \operatorname{Im}\left(\mu \bar{z}_{2}\right) j_{1} j_{2}}\right), \\
h_{4} & =\frac{\bar{\mu}\left(j_{1}+j_{2}\right) \omega_{1}}{4 \operatorname{Im}\left(\mu \bar{z}_{2}\right) j_{1} j_{2}}, \\
t_{3} & =-a_{2}=\frac{i \bar{\mu}\left(j_{1}-j_{2}\right) \omega_{1}}{4 \operatorname{Im}\left(\mu \bar{z}_{2}\right) j_{1} j_{2}} .
\end{aligned}
$$

where the volume and curvature read

$$
\begin{aligned}
\rho^{3} & =2 \epsilon_{1} \operatorname{Im}\left(\mu \bar{z}_{2}\right)\left(j_{1} j_{2}\right)^{\frac{3}{2}} \\
R_{6} & =-2|\mu|^{2}\left(2+\frac{\left(j_{1}^{2}+j_{2}^{2}\right) \omega_{1}^{2}}{4 \operatorname{Im}\left(\mu \bar{z}_{2}\right)^{2} j_{1}^{2} j_{2}^{2}}\right) .
\end{aligned}
$$

The orientifold charges are:

$$
\begin{aligned}
& N_{O 7}^{(1)}=N_{O 7}^{(2)}=-10 \epsilon_{1} e^{-\phi}|\mu|^{2} \sqrt{j_{1} j_{2}}, \\
& N_{O 5}^{(1)}=-\epsilon_{1} \frac{4 e^{-\phi}|\mu|^{2}}{\sqrt{j_{1} j_{2}} \operatorname{Im}\left(\mu \bar{z}_{2}\right) \omega_{1}}\left(5\left(j_{1} j_{2} \operatorname{Im}\left(\mu \bar{z}_{2}\right)\right)^{2}+\left(j_{1}^{2}+j_{1} j_{2}+j_{2}^{2}\right) \omega_{1}^{2}\right), \\
& N_{O 5}^{(2)}=-20 e^{-\phi} \epsilon_{1}|\mu|^{2} \sqrt{j_{1} j_{2}} \operatorname{Im}\left(\mu \bar{z}_{2}\right) \omega_{1} .
\end{aligned}
$$

\subsubsection{Model 4.1}

The solution is

$$
\begin{aligned}
z_{1} & =-2 \mu \epsilon_{1} \sqrt{-j_{1} j_{2}}, \\
\omega_{2} & =-\epsilon_{1} \sqrt{-j_{1} j_{2}},
\end{aligned}
$$




$$
\begin{aligned}
& h_{1}=\frac{i}{4} \bar{\mu}\left(2+\frac{i j_{1}}{2 \operatorname{Im}\left(\mu \bar{z}_{2}\right) \omega_{1}}\right), \\
& h_{4}=i a_{2}=i t_{3}=-\frac{\bar{\mu} j_{1}}{4 \operatorname{Im}\left(\mu \bar{z}_{2}\right) \omega_{1}} .
\end{aligned}
$$

where

$$
\begin{aligned}
& \rho^{3}=-2 \epsilon_{1} \operatorname{Im}\left(\mu \bar{z}_{2}\right)\left(-j_{1} j_{2}\right)^{\frac{3}{2}}, \\
& R_{6}=-2|\mu|^{2}\left(2+\frac{j_{1}^{2}}{4 \operatorname{Im}\left(\mu \bar{z}_{2}\right)^{2} \omega_{1}^{2}}\right),
\end{aligned}
$$

and the charges are

$$
\begin{aligned}
& N_{O 7}^{(1)}=-N_{O 7}^{(2)}=-10 \epsilon_{1} e^{-\phi}|\mu|^{2} \sqrt{-j_{1} j_{2}}, \\
& N_{O 5}^{(1)}=20 \epsilon_{1} e^{-\phi}|\mu|^{2}\left(-j_{1} j_{2}\right)^{\frac{3}{2}} \frac{\operatorname{Im}\left(\mu \bar{z}_{2}\right)}{\omega_{1}}, \\
& N_{O 5}^{(2)}=-4 \epsilon_{1} e^{-\phi}|\mu|^{2} \sqrt{-j_{1} j_{2}}\left(\frac{j_{1}^{2}+5 \operatorname{Im}\left(\mu \bar{z}_{2}\right)^{2} \omega_{1}^{2}}{\operatorname{Im}\left(\mu \bar{z}_{2}\right) \omega_{1}}\right) .
\end{aligned}
$$

\subsubsection{Model 5.1}

The solution is:

$$
\begin{aligned}
z_{1} & =-2 \mu \epsilon_{1} \sqrt{-j_{1} j_{2}}, \\
\omega_{2} & =-\epsilon_{1} \sqrt{-j_{1} j_{2}}, \\
h_{1} & =\frac{i \bar{\mu}}{2}, \\
h_{4} & =a_{2}=t_{3}=0,
\end{aligned}
$$

where

$$
\begin{aligned}
\rho^{3} & =-2 \epsilon_{1} \operatorname{Im}\left(\mu \bar{z}_{2}\right)\left(-j_{1} j_{2}\right)^{\frac{3}{2}}, \\
R_{6} & =-4|\mu|^{2}
\end{aligned}
$$

and the charges are

$$
\begin{aligned}
& N_{O 7}^{(1)}=-N_{O 7}^{(2)}=-10 \epsilon_{1} e^{-\phi}|\mu|^{2} \sqrt{-j_{1} j_{2}}, \\
& N_{O 5}^{(1)}=20 \epsilon_{1} e^{-\phi}|\mu|^{2}\left(-j_{1} j_{2}\right)^{\frac{3}{2}} \frac{\operatorname{Im}\left(\mu \bar{z}_{2}\right)}{\omega_{1}}, \\
& N_{O 5}^{(2)}=-20 \epsilon_{1} e^{-\phi}|\mu|^{2} \sqrt{-j_{1} j_{2}} \operatorname{Im}\left(\mu \bar{z}_{2}\right) \omega_{1} .
\end{aligned}
$$

This solution can be obtained from a T-duality of the $\mathrm{O} 6$ toroidal orientifold in massive IIA SUGRA. 


\section{Discussion}

In this paper we have studied what are the general conditions to have 10-dimensional $\mathcal{N}=1 \mathrm{AdS}_{4}$ vacua from IIB compactifications on smeared $\mathrm{O5} / \mathrm{O} 7$ orientifolds. The orientifold projections forces the internal manifold to be of $\mathrm{SU}(2)$ structure. We give the supersymmetry conditions both in terms of the pure spinors defined on the internal manifold [16] and in terms of $\mathrm{SU}(2)$ torsion classes. Our analysis is completely 10-dimensional and parallels the existing results for solutions in type IIA [7].

The main purpose of the paper was to look for possible candidates for scale separation in purely classical type IIB backgrounds. It is natural to look first for relatively simple classes of manifolds, whose geometry is under control, namely reductive cosets and nilmanifolds. We applied our general formalism to such manifolds and we checked whether the vacua we found admit limits where the cosmological constant and the string coupling are small, while the internal volume is large. We also checked scale separation, using both criteria we discussed in section 4. Our results are summarised in table 2. Clearly, in order to have a complete prove that scale separation is indeed possible, one should compute the Kaluza-Klein spectrum. It is nonetheless promising that some of the vacua survive a first analysis based on scaling arguments. It would also be interesting to have a better understanding of when and why the different scaling criteria have to agree or not.

There are various issues that call for further research. Most pressing is the status of the orientifold sources. Apart from the charge quantisation, which has not been worked out, it is essential to understand how to localise the orientifold planes. Likely this question is more tractable using the pure spinor formalism, as was attempted for localised O6 solutions in massive IIA [13]. If an analogy can be made with solutions that feature parallel orientifolds $[16,17]$ then one can expect that localisation will change the geometry but that the very existence of the solution is not invalidated. It should also be possible to find solutions of our type (IIB SU(2) structures) without any sources, which should be relevant for holography. We have not been able to do this for the class of manifolds we considered (reductive cosets and nil manifolds. ${ }^{11}$ ), but it would be interesting to look for instance at Solv manifolds. It is perhaps even more relevant to break supersymmetry and to look for non-SUSY AdS vacua in this context or even dS vacua. In IIA meta-stable non-SUSY AdS vacua have been found using Ansatze that are close to that of the SUSY AdS solutions [10]. Interestingly, the same has been done for dS solutions $[36,44,51,52],{ }^{12}$ although none of the latter examples turned out meta-stable. Clearly more examples are required and the results in this paper could offer a first step towards achieving this. Already a (unstable) de Sitter critical point was numerically found in [28]. It would be worthwhile to verify whether this numerical solution lifts to a simple 10-dimensional solution. Finally we hope that some of the $\mathrm{SU}(2)$ structure technology we developed in this paper has its applications to $\mathrm{SU}(2)$ structure solutions in other contexts, such as in IIA, see for instance [55].

\footnotetext{
${ }^{11}$ This is obvious for nil manifolds as they are negatively curved whereas source less solutions require positively curved internal spaces [46].

${ }^{12}$ These de Sitter constructions are in part inspired on the proposals in [53, 54].
} 


\section{Acknowledgments}

We would like to thank U. Danielsson, A. Tomasiello, D. Tsimpis, T. Wrase and A. Zaffaroni for useful discussions. TVR is supported by a Pegasus Marie Curie fellowship of the FWO. MP would like to thank the Theory Group at Imperial College for the kind hospitality during the final part of this project.

\section{A Conditions for $\mathrm{AdS}_{4}$ vacua with $\mathcal{N}=1$ SUSY in type IIB SUGRA}

In this appendix we derive the general conditions that the fluxes and the internal geometry must satisfy in order to have four-dimensional AdS vacua with $\mathcal{N}=1$ supersymmetry in type IIB supergravity.

We consider ten-dimensional geometries that are (warped) products of fourdimensional Anti-de Sitter space times a compact six-dimensional internal manifold, $Y$, and we allow for non-trivial fluxes that do not break the maximal symmetry of $\mathrm{AdS}_{4}$. We also restrict our attention to vacua with $\mathcal{N}=1$ supersymmetry. Backgrounds of this kind can be determined simply by imposing the supersymmetry variations and the Bianchi identities for the fluxes. ${ }^{13}$ To analyse the supersymmetry variations we will use the formalism of Generalised Complex Geometry [34, 35]. The idea is that the ten-dimensional supersymmetry conditions can be rewritten as a set of differential equations on globally defined forms on the internal manifold [30].

\section{A.1 SU(3) and SU(2) structures}

In this section we present some basic definitions and identities for $\mathrm{SU}(3)$ and $\mathrm{SU}(2)$ structures with the purpose of fixing conventions and providing the necessary tools to follow the derivations in the paper.

In type IIB $\mathcal{N}=1$ compactifications to a maximally symmetric four-dimensional manifold the ten-dimensional supersymmetry parameters factorise as

$$
\epsilon_{i}=\zeta_{+} \otimes \eta_{+}^{i}+\zeta_{-} \otimes \eta_{-}^{i} \quad i=1,2
$$

where $\zeta_{+}$and $\eta_{+}^{i}$ are Weyl spinors in four and six dimensions respectively, and $\eta_{-}^{i}=\left(\eta_{+}^{i}\right)^{*}$ and $\zeta_{-}=\left(\zeta_{+}\right)^{*}$.

We assume that the spinors $\eta_{1}$ and $\eta_{2}$ are globally defined. This means that the structure group of $Y$ is reduced to a subgroup $G \in \mathrm{SO}(6)$. What $G$ is depends on the relation between the two spinors, $\eta_{i}$, which generically $\eta_{+}^{1}$ and $\eta_{+}^{2}$ are neither parallel nor orthogonal. We can parametrise them as

$$
\begin{aligned}
& \eta_{+}^{1}=a \eta_{+} \\
& \eta_{+}^{2}=b\left(k_{\|} \eta_{+}+\frac{1}{2} k_{\perp} z_{m} \gamma^{m} \eta_{-}\right)
\end{aligned}
$$

\footnotetext{
${ }^{13}$ For a space-time which is a warped product $M_{d} \times Y_{10-d}$ it can be shown that the supersymmetry equations plus flux Bianchi identities imply the full set of equations of motions.
} 
where $z=z_{m} \gamma^{m}$ is a six-dimensional one-form, $\eta_{+}$is a globally defined Weyl spinor of norm one and $k_{\|}^{2}+k_{\perp}^{2}=1$. When $k_{\|}=1$ and $k_{\perp}=0$ the internal manifold is said to be of $\mathrm{SU}(3)$ structure, in the opposite case $k_{\|}=0$ and $k_{\perp}=1$ the structure is $\mathrm{SU}(2)$, while the general case is often referred to as dynamical $\mathrm{SU}(2)$ structure. $a$ and $b$ are two complex functions determining the norm of $\eta^{1}$ and $\eta^{2}$

$$
\eta^{1 \dagger} \eta^{1}=|a|^{2} \quad \eta^{2 \dagger} \eta^{2}=|b|^{2} .
$$

An equivalent definition of a $G$-structure is given in terms of invariant forms on the manifold, which can be constructed as bilinears in the internal spinors. For SU(3) these are a real two-form and a holomorphic three-form

$$
\begin{aligned}
J_{m n} & =-i \eta_{+}^{\dagger} \gamma_{m n} \eta_{+}, \\
\Omega_{m n p} & =-i \eta_{-}^{\dagger} \gamma_{m n p} \eta_{+},
\end{aligned}
$$

satisfying

$$
J \wedge \Omega=0, \quad J \wedge J \wedge J=\frac{3}{4} i \Omega \wedge \bar{\Omega},
$$

and

$$
\begin{aligned}
& * \Omega=-i \Omega, \\
& * J=-\frac{1}{2} J^{2} .
\end{aligned}
$$

The volume of the internal manifold is given by $J^{3}=-6 \operatorname{vol}_{6}$.

For the $\mathrm{SU}(2)$ structure, we have a complex one-form, a real and a holomorphic two-form

$$
\begin{aligned}
z_{m} & =-\chi_{-}^{\dagger} \gamma_{m} \eta_{+}, \\
j_{m n} & =-i \eta_{+}^{\dagger} \gamma_{m n} \eta_{+}+i \chi_{+}^{\dagger} \gamma_{m n} \chi_{+}, \\
\omega_{m n} & =-i \chi_{+}^{\dagger} \gamma_{m n} \eta_{+},
\end{aligned}
$$

where $\chi_{+}=\frac{1}{2} z \eta_{-}$satisfying

$$
\begin{aligned}
z\llcorner\bar{z} & =2, \quad z\llcorner z=\bar{z}\llcorner\bar{z}=0, \\
j \wedge \omega & =0, \\
z\llcorner j & =z\llcorner\omega=0, \\
j \wedge j & =\frac{1}{2} \omega \wedge \bar{\omega} .
\end{aligned}
$$

The one-form $z$ provides an almost product structure on $Y$, defined locally by

$$
R_{m}^{n}=z_{m} \bar{z}^{n}+\bar{z}_{m} z^{n}-\delta_{m}^{n}, \quad m, n=1, \ldots, 6,
$$

which induces a (global) decomposition of the tangent space in

$$
T M=T_{2} M \oplus T_{4} M .
$$

Notice that the sub-bundle $T_{2} M$ is spanned by the real and imaginary parts of the form $z$. 


\section{A.1.1 Torsion classes}

The forms defining a $G$-structure are generically not closed. Their differential can be decomposed into representations of the structure group $G$, the so called torsion classes, and we classify the different $G$-structures depending on which torsion class is non-zero $[39,40]$.

For $\mathrm{SU}(3)$ structure such decomposition is very simple

$$
\begin{aligned}
\mathrm{d} J & =\frac{3}{2} \operatorname{Im}\left(\bar{W}_{1} \Omega\right)+W_{4} \wedge J+W_{3}, \\
\mathrm{~d} \Omega & =W_{1} \wedge J \wedge J+W_{2} \wedge J+\bar{W}_{5} \wedge \Omega,
\end{aligned}
$$

where $W_{1}$ is a complex scalar which is a singlet of $\mathrm{SU}(3), W_{2}$ is a complex primitive $(1,1)$ form transforming in the adjoint, $W_{3}$ a real primitive $(2,1)$ and $(1,2)$ form in the $\mathbf{6} \oplus \overline{\mathbf{6}}, W_{4}$ a real vector and $W_{5}$ a complex $(1,0)$-form in the $\mathbf{3} \oplus \overline{\mathbf{3}}$.

The same expansion for $\mathrm{SU}(2)$ structures is more involved since the torsion classes are now 20: 8 complex singlet $S_{i}, 8$ complex doublets $V_{i}$ and 4 complex triplets $T_{i}$. We have ${ }^{14}$

$$
\begin{aligned}
\mathrm{d} z= & S_{1} \omega+S_{2} j+S_{3} z \wedge \bar{z}+S_{4} \bar{\omega}+z \wedge\left(V_{1}+\bar{V}_{2}\right)+\bar{z} \wedge\left(V_{3}+\bar{V}_{4}\right)+T_{1}, \\
\mathrm{~d} j= & S_{5} \bar{z} \wedge \omega+S_{6} z \wedge \omega+\frac{1}{2}\left(S_{7}+\bar{S}_{8}\right) z \wedge j+j \wedge V_{5}+z \wedge \bar{z} \wedge V_{6}+z \wedge T_{2}+\text { c.c. }, \\
\mathrm{d} \omega= & S_{7} z \wedge \omega+S_{8} \bar{z} \wedge \omega-2 \bar{S}_{5} z \wedge j-2 \bar{S}_{6} \bar{z} \wedge j+i z \wedge \bar{z} \wedge\left(\bar{V}_{6}\llcorner\omega)+j \wedge\left(V_{7}+\bar{V}_{8}\right)\right. \\
& +z \wedge T_{3}+\bar{z} \wedge T_{4},
\end{aligned}
$$

where the relations between the representations in $\mathrm{d} j$ and $\mathrm{d} \omega$ are implied by the conditions $\mathrm{d}(j \wedge \omega)=\mathrm{d}(j \wedge j)=\mathrm{d}(\omega \wedge \omega)=0$.

Notice that the doublet $V_{i}$ are holomorphic vectors with respect to the complex structure defined by $j$,

$$
\omega \wedge V_{i}=0
$$

while the $T_{i}$ are $(1,1)$ and primitive

$$
j \wedge T_{i}=\omega \wedge T_{i}=0
$$

and can be decomposed on the basis of anti-self dual two-forms $\tilde{j}_{1}, \tilde{j}_{2}, \tilde{j}_{3}$ transforming in the 3 of $\mathrm{SU}(2)$

$$
T_{i}=\sum_{a=1}^{3} t_{a}^{i} \tilde{j}_{a} .
$$

\section{A.2 Conditions for $\mathrm{AdS}_{4}$ vacua}

$G$-structures have been successfully applied to flux compactifications as tools to classify the internal manifolds allowing for SUSY flux vacua. The idea is to rewrite the supersymmetry variations as differential equations on the $G$-invariant forms, then susy solutions are obtained equating fluxes and torsions in the appropriate representations. In particular, for four-dimensional flux compactifications, the relevant structures are $\mathrm{SU}(3)$ and $\mathrm{SU}(2)$.

\footnotetext{
${ }^{14}$ A simple way to deduce such classes is to decompose the $\mathrm{SU}(3)$ torsion classes according to $\mathrm{SU}(3) \rightarrow$ $\mathrm{SU}(2) \times \mathrm{U}(1)$. We added two vector representations in $\mathrm{d} z$ that were missing in [41].
} 
Generalised Complex Geometry [34,35] is a further refinement of this approach which allows for a unified treatment of all flux vacua with a given amount of supersymmetry, encompassing in such a way all possible $G$-structures.

In this paper we focus on $\mathcal{N}=1$ supersymmetry. As shown in [30], the ten-dimensional supersymmetry conditions can be rewritten as a set of differential equations on a pair of globally defined poly-forms on $Y$. Such polyforms, or pure spinors, are constructed by tensoring the two supersymmetry parameters on the internal manifold $\eta^{1}$ and $\eta^{2}$

$$
\Phi_{ \pm}=\eta_{+}^{1} \otimes \eta_{ \pm}^{2 \dagger}
$$

The explicit expression for $\Phi_{ \pm}$depends on the relation between $\eta_{+}^{1}$ and $\eta_{+}^{2}$. In the general case (A.2), they are defined as

$$
\begin{aligned}
& \Phi_{-}=-\frac{a b}{8} z \wedge\left(k_{\perp} e^{-i j}+i k_{\|} \omega\right), \\
& \Phi_{+}=\frac{a \bar{b}}{8} e^{z \bar{z} / 2}\left(k_{\|} e^{-i j}-i k_{\perp} \omega\right),
\end{aligned}
$$

where $z, j$ and $\omega$ are the $\mathrm{SU}(2)$ structure forms (A.8). For the special cases of $\mathrm{SU}(3)$ structure $\left(k_{\|}=1, k_{\perp}=0\right)$ and rigid $\mathrm{SU}(2)$ structure $\left(k_{\|}=0, k_{\perp}=1\right)$ the pure spinors reduce to

$$
\begin{aligned}
& \Phi_{-}=-i \frac{a b}{8} \Omega, \\
& \Phi_{+}=\frac{a \bar{b}}{8} e^{-i J},
\end{aligned}
$$

where $\Omega$ and $J$ are the $\mathrm{SU}(3)$ invariant forms defining the $\mathrm{SU}(3)$ structure, (A.4), and

$$
\begin{aligned}
& \Phi_{-}=-\frac{a b}{8} z \wedge e^{-i j}, \\
& \Phi_{+}=-i \frac{a \bar{b}}{8} e^{z \bar{z} / 2} \omega,
\end{aligned}
$$

respectively.

For type IIB compactifications to $\mathrm{AdS}_{4}$ the ten-dimensional supersymmetry variations are equivalent to the following set of equations on the pure spinors $\Phi_{ \pm}[30]$

$$
\begin{aligned}
(\mathrm{d}-H \wedge)\left(e^{2 A-\phi} \Phi_{-}\right) & =-2 \mu e^{A-\phi} \operatorname{Re} \Phi_{+}, \\
(\mathrm{d}-H \wedge)\left(e^{A-\phi} \operatorname{Re} \Phi_{+}\right) & =0, \\
(\mathrm{~d}-H \wedge)\left(e^{3 A-\phi} \operatorname{Im} \Phi_{+}\right) & =-3 e^{2 A-\phi} \operatorname{Im}\left(\bar{\mu} \Phi_{-}\right)-\frac{1}{8} e^{4 A} * \lambda(F),
\end{aligned}
$$

where $\phi$ is the dilaton, $A$ the warp factor and $F$ is the sum of the RR field strength on $Y$, $F=F_{1}+F_{3}+F_{5} . \lambda$ is the transposition on a form

$$
\lambda\left(F_{k}\right)=(-)^{[k / 2]} F_{k} .
$$

It can also be shown [30] that for $\mathrm{AdS}_{4}$ vacua supersymmetry also requires the norms of the two six-dimensional spinors to be equal

$$
\left\|\eta_{+}^{1}\right\|^{2}=\left\|\eta_{+}^{2}\right\|^{2} \quad \Rightarrow \quad|a|^{2}=|b|^{2}=e^{A} .
$$


In the following we set

$$
a=\bar{b}, \quad b=a e^{-i \theta} .
$$

Plugging the explicit form of (2.9) and (A.28), into the susy variations (2.2)-(2.4), one can deduce equations of definite form degree for the forms $z, \omega$ and $j$ and the fluxes. These give a set of general conditions for $\mathrm{AdS}_{4} \mathcal{N}=1$ susy vacua.

\section{A.3 No $\mathrm{AdS}_{4}$ vacua for $\mathrm{SU}(3)$ structure}

From equation (2.2) it is immediate to see that it is not possible to have $\mathrm{AdS}_{4}$ vacua with $\mathrm{SU}(3)$ structure. Indeed, in this case the $\Phi_{-}$only contains a three-form term, so that one has to zero the zero- and two-form terms in $\operatorname{Re} \Phi_{+}$, which for $k_{\perp}=0$, give

$$
\begin{aligned}
\cos \theta & =0, \\
\sin \theta\left(j+\frac{i}{2} z \wedge \bar{z}\right) & =0 .
\end{aligned}
$$

Clearly these two equations cannot be solve at the same time.

\section{A.4 $\mathrm{AdS}_{4}$ vacua for $\mathrm{SU}(2)$ structures}

Let us consider then the most general pure spinors defined in (A.23) and (A.24), and first expand (A.29)

$$
(\mathrm{d}-H \wedge)\left(e^{2 A-\phi} \Phi_{-}\right)=-2 \mu e^{A-\phi} \operatorname{Re} \Phi_{+} .
$$

The zero-form component gives

$$
\mu k_{\|} \cos \theta=0,
$$

which implies

$$
k_{\|}=0 \quad \text { or } \quad \cos \theta=0 .
$$

The first choice corresponds to a rigid $\mathrm{SU}(2)$ structure, while the second fixes the relative phase of $a$ and $b$.

\section{A.4.1 Rigid SU(2) structures}

Fixing the phase of $a$ and $b$ is equivalent to fixing the orientifold projection. To allow for both $\mathrm{O} 5$ and $\mathrm{O} 7$ planes, as we need in this paper, we have to set $k_{\|}=0$ and concentrate on rigid $\mathrm{SU}(2)$ structure

$$
k_{\|}=0 \quad k_{\perp}=1 .
$$

It is convenient to define the new two-form

$$
\hat{\omega}=e^{i \theta} \omega .
$$

With this redefinition, the two-, four- and six-form components of (A.29) give

$$
\begin{aligned}
\mathrm{d}\left(e^{3 A-\phi} z\right) & =2 \mu e^{2 A-\phi} \operatorname{Im} \hat{\omega} \\
\mathrm{d}\left(e^{3 A-\phi} z \wedge j\right) & =i e^{2 A-\phi} H \wedge z+e^{2 A-\phi} \mu z \wedge \bar{z} \wedge \operatorname{Re} \hat{\omega} \\
\mathrm{d}\left(e^{3 A-\phi} z \wedge j \wedge j\right) & =2 i e^{3 A-\phi} H \wedge \hat{z} \wedge j
\end{aligned}
$$


Plugging (A.41) in (A.42) and recalling that $\operatorname{Im} \omega \wedge j=0$ for an $\mathrm{SU}(2)$ structure we obtain

$$
z \wedge\left(\mathrm{d} j-i H+\mu e^{-A} \bar{z} \operatorname{Re} \hat{\omega}\right)=0 .
$$

It is also straightforward to show that (A.43) is implied by (A.42). Indeed substituting (A.42) in (A.43) gives

$$
z \wedge j \wedge(\mathrm{d} j-i H)=0
$$

which is a consequence of (A.44).

Let us now consider the second equation, (A.30),

$$
(\mathrm{d}-H \wedge)\left(e^{A-\phi} \operatorname{Re} \Phi_{+}\right)=0 .
$$

Expanded in forms it gives a three- and five-form equation

$$
\begin{aligned}
\mathrm{d}\left(e^{2 A-\phi} \operatorname{Im} \hat{\omega}\right) & =0 \\
\mathrm{~d}\left(e^{2 A-\phi} z \wedge \bar{z} \wedge \operatorname{Re} \hat{\omega}\right) & =2 i e^{2 A-\phi} H \wedge \operatorname{Im} \hat{\omega}
\end{aligned}
$$

Finally we have to expand (A.31)

$$
(\mathrm{d}-H \wedge)\left(e^{3 A-\phi} \operatorname{Im} \Phi_{+}\right)=-3 e^{A-\phi} \operatorname{Im}\left(\bar{\mu} \Phi_{-}\right)-\frac{1}{8} e^{4 A} * \lambda(F) .
$$

This gives

$$
\begin{aligned}
* F_{5}= & 3 e^{-A-\phi} \operatorname{Im}(\bar{\mu} z), \\
* F_{3}= & -e^{-4 A} \mathrm{~d}\left(e^{4 A-\phi} \operatorname{Re} \hat{\omega}\right)+3 e^{-A-\phi} \operatorname{Re}(\bar{\mu} z) \wedge j, \\
* F_{1}= & -i \mathrm{~d}(2 A-\phi) z \wedge \bar{z} \wedge \operatorname{Im} \hat{\omega}-e^{-\phi} H \wedge \operatorname{Re} \hat{\omega} \\
& +\frac{1}{2} e^{-A-\phi} \operatorname{Im}(\bar{\mu} z) \wedge j \wedge j,
\end{aligned}
$$

where in the last equation we used (A.47). In summary the non trivial susy conditions are

$$
\begin{aligned}
\mathrm{d}\left(e^{3 A-\phi} z\right) & =2 \mu e^{2 A-\phi} \operatorname{Im} \hat{\omega} \\
z \wedge\left(\mathrm{d} j-i H+\mu e^{-A} z \wedge \operatorname{Re} \hat{\omega}\right) & =0 \\
\mathrm{~d}\left(e^{2 A-\phi} \operatorname{Im} \hat{\omega}\right) & =0 \\
\mathrm{~d}\left(e^{2 A-\phi} z \wedge \bar{z} \wedge \operatorname{Re} \hat{\omega}\right) & =2 i e^{2 A-\phi} H \wedge \operatorname{Im} \hat{\omega}
\end{aligned}
$$

plus equations (A.50)-(A.52) for the fluxes.

To make contact with previous literature, we can express the equations above using the $\mathrm{SU}(2)$ intrinsic torsions (A.18). The idea is to decompose all the objects in the equations in representations of $\mathrm{SU}(2)$ and then obtain a set of conditions for the fields in the various representations. To decompose the exterior derivatives we use the torsion classes defined in (A.18), while for the fluxes we have

$$
\begin{aligned}
& H=h_{1} z \wedge \hat{\omega}+h_{2} \bar{z} \wedge \hat{\omega}+h_{3} z \wedge j+z \wedge \bar{z} \wedge h_{1}^{(2)}+h_{2}^{(2)} \wedge j+z \wedge h^{(3)}+\text { c.c. } \\
& F_{1}=f_{1} z+f_{1}^{(2)}+\text { c.c. }
\end{aligned}
$$




$$
\begin{aligned}
& F_{3}=f_{2} z \wedge \hat{\omega}+f_{3} \bar{z} \wedge \hat{\omega}+f_{4} z \wedge j+z \wedge \bar{z} \wedge f_{2}^{(2)}+f_{3}^{(2)} \wedge j+z \wedge f^{(3)}+\text { c.c. } \\
& F_{5}=f_{5} z \wedge j \wedge j+z \wedge \bar{z} \wedge j \wedge f_{4}^{(2)}+\text { c.c. }
\end{aligned}
$$

where $h_{i}$ and $f_{i}$ are complex scalars in the singlet representation of $\mathrm{SU}(2), h_{i}^{(2)}$ and $f_{i}^{(2)}$ are holomorphic vectors in the $\mathbf{2}$ and $h^{(3)}$ and $f^{(3)}$ are complex two forms in the triplet representation, which are $(1,1)$ and primitive with respect to $j$.

For completeness we also give the decomposition of Hodge dual fluxes

$$
\begin{aligned}
* H= & -i h_{1} z \wedge \hat{\omega}+i h_{2} \bar{z} \wedge \hat{\omega}-i h_{3} z \wedge j-i z \wedge *_{4} h^{(3)}+2 i *_{4} h_{1}^{(2)} \\
& -\frac{i}{2} z \wedge \bar{z}\left(h_{2}^{(2)}\llcorner j)+\text { c.c. },\right. \\
* F_{1}= & -\frac{i}{2} f_{1} z \wedge j \wedge j-\frac{i}{2} z \wedge \bar{z} \wedge *_{4} f_{1}^{(2)}+\text { c.c. }, \\
* F_{3}= & -i f_{2} z \wedge \hat{\omega}+i f_{3} \bar{z} \wedge \hat{\omega}-i f_{4} z \wedge j-i z \wedge *_{4} f^{(3)}+2 i *_{4} f_{2}^{(2)} \\
& -\frac{i}{2} z \wedge \bar{z} \wedge\left(f_{3}^{(2)}\llcorner j)+\text { c.c. },\right. \\
* F_{5}= & -2 i f_{5} z+2 i f_{4}^{(2)}\llcorner j+\text { c.c. },
\end{aligned}
$$

where we used the fact that a product structure allows to split $*_{6}=*_{2} *_{4}$ and $*_{4}(v \wedge \xi)=(-1)^{\operatorname{deg} \xi} v\left\llcorner *_{4} \xi\right.$.

We can now look at the SUSY variations. Let us first consider (A.53)-(A.56). We find that the singlets in the torsions must satisfy

$$
\begin{array}{rlrl}
S_{2}=0, & S_{1} & =-S_{4}=-i \mu e^{-A}, \\
S_{3}=\frac{1}{2} \partial_{\bar{z}}(3 A-\phi), & S_{5} & =\bar{S}_{6}=i \bar{h}_{1}-\frac{1}{2} e^{-A} \mu, \\
S_{7} & =\bar{S}_{8}=-\frac{1}{2} \partial_{z}(2 A-\phi) .
\end{array}
$$

Similarly, there are conditions on the vectors

$$
\begin{array}{ll}
V_{3}=V_{4}=V_{6}=0, & V_{7}=i\left(\bar{\partial}_{4} A+\bar{h}_{1}^{(2)}\right)\llcorner\omega, \\
V_{5}=i h_{2}^{(2)}, & V_{8}=i\left[\bar{\partial}_{4}(3 A-\phi)+\bar{h}_{1}^{(2)}\right]\llcorner\omega, \\
V_{1}=V_{2}=\partial_{4}(3 A-\phi), &
\end{array}
$$

and the two-forms

$$
T_{1}=0, \quad T_{2}=-i h^{(3)}, \quad T_{3}=\bar{T}_{4},
$$

and the NS flux singlets

$$
h_{1}=\bar{h}_{2}, \quad h_{3}=-\frac{i}{2} \partial_{z}(2 A-\phi) .
$$


Finally the equations (A.50)-(A.52) for the RR fluxes give

$$
\begin{array}{rlrl}
f_{1} & =-e^{-\phi}\left(4 i h_{1}-\frac{1}{2} \bar{\mu} e^{-A}\right), f_{1}^{(2)} & =i e^{-\phi} \omega\left\llcorner\left[\bar{\partial}_{4}(2 A-\phi)+\bar{h}_{1}^{(2)}\right],\right. \\
f_{2} & =\bar{f}_{3}=-\frac{i}{2} e^{-\phi} \partial_{z} A, & f_{2}^{(2)} & =\frac{i}{2} e^{-\phi} \omega\left\llcorner\left[\bar{\partial}_{4}(4 A-\phi)-\bar{h}_{1}^{(2)}\right],\right. \\
f_{4} & =\frac{1}{2} e^{-\phi}\left(4 h_{1}+i \bar{\mu} e^{-A}\right) & f_{3}^{(2)} & =f_{4}^{(2)}=0, \\
f_{5} & =\frac{3}{4} e^{-A-\phi} \bar{\mu} & f^{(3)} & =i e^{-\phi} T_{3} .
\end{array}
$$

The conditions coming from the Bianchi identities are too involved to give for a generic SU(2) structure. They become more amenable in presence of O-planes, since the orientifold projections considerably restrict the allowed torsion classes. We discuss the case of parallelisable manifolds in the main text.

\section{B Type IIA $\mathrm{AdS}_{4}$ solutions and separation of scales}

The best known examples of $\mathrm{AdS}_{4}$ compactifications and, among them, $\mathrm{AdS}_{4}$ vacua with separation of scales are in type IIA. In this appendix we first remind the general conditions for $\mathcal{N}=1 \mathrm{AdS}_{4}$ vacua in type type IIA [7] and then discuss separation of scales for a specific example.

\section{B.1 Lust-Tsimpis SU(3)-structures}

The general conditions for $\mathrm{AdS}_{4}$ with $\mathcal{N}=1$ supersymmetry on $\mathrm{SU}(3)$ structure manifolds given in [7] is easily derived using the pure spinor equations [16].

In type IIA the supersymmetry variations take the same form as in (A.29)-(A.31) with $\Phi_{+}$and $\Phi_{-}$exchanged

$$
\begin{aligned}
(\mathrm{d}-H \wedge)\left(e^{2 A-\phi} \Phi_{+}\right) & =-2 \mu e^{A-\phi} \operatorname{Re} \Phi_{-}, \\
(\mathrm{d}-H \wedge)\left(e^{A-\phi} \operatorname{Re} \Phi_{-}\right) & =0 \\
(\mathrm{~d}-H \wedge)\left(e^{3 A-\phi} \operatorname{Im} \Phi_{-}\right) & =-3 e^{2 A-\phi} \operatorname{Im}\left(\bar{\mu} \Phi_{+}\right)-\frac{1}{8} e^{4 A} * \lambda(F) .
\end{aligned}
$$

For an $\mathrm{SU}(3)$ structure, the pure spinors have the form (2.14)

$$
\Phi_{-}=-\frac{i a b}{8} \Omega \quad \Phi_{+}=\frac{a \bar{b}}{8} e^{-i J}
$$

where we set $\mathrm{e}^{A / 2}=|a|=|b|=1$. It is convenient to define

$$
\bar{a} b \mu \equiv \hat{\mu} \equiv m+i \tilde{m}, \quad \hat{\Omega}=-i a b \Omega,
$$

where number $m$ is not to be confused with the Romans mass, although it will turn out to be proportional to it. Then real and imaginary parts of the pure spinors can be written as

$$
\begin{aligned}
& 8 \operatorname{Re}\left(\bar{\mu} \Phi_{+}\right)=m-\tilde{m} J-m \frac{1}{2} J^{2}+\tilde{m} \frac{1}{3 !} J^{3}, \\
& 8 \operatorname{Im}\left(\bar{\mu} \Phi_{+}\right)=-\tilde{m}-m J+\tilde{m} \frac{1}{2} J^{2}+m \frac{1}{3 !} J^{3},
\end{aligned}
$$




$$
\begin{aligned}
& 8 \operatorname{Re}\left(\Phi_{-}\right)=\hat{\Omega}_{R}, \\
& 8 \operatorname{Im}\left(\Phi_{-}\right)=\hat{\Omega}_{I} .
\end{aligned}
$$

The supersymmetry variations (B.1) and (B.2) imply

$$
H=2 m \hat{\Omega}_{R}, \quad \mathrm{~d} J=2 \tilde{m} \hat{\Omega}_{R} .
$$

This leads to the following restrictions on the torsions

$$
\begin{aligned}
W_{1} & =-\frac{4}{3} i \tilde{m}, & W_{4} & =0, \\
\operatorname{Re}\left(W_{2}\right) & =0, & \operatorname{Re}\left(\bar{W}_{5} \hat{\Omega}\right) & =0 .
\end{aligned}
$$

The last pure spinor equation, (B.3) defines the RR field strengths

$$
\begin{aligned}
\mathrm{e}^{\phi} F_{0} & =5 m, \\
\mathrm{e}^{\phi} F_{2} & =\frac{\tilde{m}}{3} J+i W_{2}, \\
\mathrm{e}^{\phi} F_{4} & =\frac{3}{2} m J \wedge J, \\
\mathrm{e}^{\phi} F_{6} & =3 \tilde{m} \mathrm{vol}_{6} .
\end{aligned}
$$

The Bianchi identity for $F_{4}$ implies that $\hat{\Omega}_{R} \wedge * \operatorname{Im}\left(\bar{W}_{5} \hat{\Omega}\right)=0$, where we made use of $* W_{2}=J \wedge W_{2}$. Together with $\operatorname{Re}\left(\bar{W}_{5} \hat{\Omega}\right)=0$ we find $W_{5}=0$. The $F_{2}$ Bianchi identity $\mathrm{d} F_{2}=F_{0} H+j$ gives an equation for the source form

$$
\mathrm{e}^{\phi} j_{\mathrm{O} 6}=i \mathrm{~d} W_{2}+\left(\frac{2}{3} \tilde{m}^{2}-10 m^{2}\right) \hat{\Omega}_{R}
$$

Source-less solutions require

$$
i \mathrm{~d} W_{2}=c \Omega_{R}, \quad c=10 m^{2}-\frac{2}{3} \tilde{m}^{2} .
$$

One can easily prove that if $i \mathrm{~d} W_{2}=c \Omega_{R}$ we must have that ${ }^{15}$

$$
c=-\frac{1}{8}\left(W_{2}^{I}\right)_{a b}\left(W_{2}^{I}\right)^{a b} .
$$

Explicit manifolds for which these conditions can be fulfilled have been constructed [33].

For simplicity we restrict to the class of the solutions that obey $i \mathrm{~d} W_{2}=c \Omega_{R}$. That class has the nice property that it connects to the source-free case for special value of $c$ given in (B.17). The source form $j$ that appears in the Bianchi identity is then proportional to $\Omega_{R}$

$$
e^{\phi} j_{\mathrm{O} 6}=Q \Omega_{R},
$$

where $Q$ relates to the $\mathrm{O} 6$ or D6 charge. We now follow the argument of [11]. If we use that $R_{6}=\frac{15}{2}\left|W_{1}\right|^{2}-\frac{1}{2}\left|W_{2}\right|^{2}$, we can derive that

$$
r=\frac{6 \tilde{m}^{2}+10 m^{2}}{m^{2}+\tilde{m}^{2}}+\frac{Q}{m^{2}+\tilde{m}^{2}} .
$$

The first term is clearly bounded, so scale separation then indeed requires non-zero orientifold charge and small cosmological constant.

\footnotetext{
${ }^{15}$ This is done by considering the identity $\mathrm{d}\left(\Omega_{I} \wedge W_{2}\right)=0$ and then using the various $\mathrm{SU}(3)$-structure identities on this.
} 


\section{B.2 The rectangular torus solution}

A well known example where the conditions for a classical $\mathrm{AdS}_{4}$ vacuum with scale separation are satisfied is provided by the model of [6]: a compactification on an orbifold of $T^{6}$ with non-zero $F_{0}, F_{4}, H$-fluxes and $\mathrm{O} 6$ sources. While the $H$ and $F_{0}$ flux are constrained by the tadpole condition to be of order one, the $F_{4}$ flux is an unbounded flux quantum. All moduli are stabilised and, by scaling up the $F_{4}$ flux quanta, the conditions for scale separation can be met. Since $F_{4}$ is unbounded, there is actually an infinite number of solutions. Since the internal space has no curvature only criterium (4.20), but not (4.21) can be used.

In order to simplify things we describe the solution on the rectangular torus, by which we mean that only the dependence on the volume modulus is shown since all other moduli are fixed to their miniumum for which the torus is straight. The solution is given by

$$
\begin{aligned}
J & =\rho^{2}\left[e^{12}+e^{34}+e^{56}\right], \\
\Omega & =\rho^{3}\left(i e^{1}+e^{2}\right) \wedge\left(i e^{3}+e^{4}\right) \wedge\left(i e^{5}+e^{6}\right), \\
\operatorname{vol}_{6} & =-\rho^{6} e^{123456},
\end{aligned}
$$

where $e^{i}=\mathrm{d} x^{i}$ with the $x^{i}$ the local Cartesian coordinates on a torus. All torsion classes are zero. The $10 \mathrm{D}$ solution is given by

$$
\begin{aligned}
e^{\phi} F_{0} & =5 m, \\
e^{\phi} F_{4} & =\frac{3}{2} m J \wedge J, \\
H & =2 m \Omega_{R}, \\
e^{-\phi} j_{O 6} & =-10 m^{2} \Omega_{R}
\end{aligned}
$$

The cosmological constant is given by $V=-6 m^{2} M_{p}^{2}$.

Now we discuss the scalings and the limit to weak coupling, large volume, small cc, scale separation and we take into account flux and charge quantisation. We introduce a parameter $\lambda$ which is supposed to go to infinity

$$
\lambda \rightarrow \infty .
$$

Small cc can be achieved by making $m$ scale as follows

$$
m \rightarrow \lambda^{-1}
$$

The quantisation of Romans mass $F_{0}=n_{0}$ and the $F_{2}$ tadpole condition

$$
n_{0} h=\text { number of O-planes }
$$

imply that both Romans mass quantum and the $H$ flux quantum $h$ are bounded to be order one. We then find, from the expression of $F_{0}$ that

$$
e^{\phi} \rightarrow \lambda^{-1} \text {. }
$$


This ensures weak coupling. The boundedness of the $h$ flux quantum together with the explicit expression for $H$ flux implies that

$$
\rho \sim \lambda^{1 / 3}
$$

This brings us to large volume. Flux quantisation for $F_{4}$

$$
F_{4}=n_{4}(\text { wedges of e's })
$$

implies that $n_{4}$ will become a large flux quantum

$$
n_{4} \sim \lambda^{2 / 3} .
$$

Note that the $F_{4}$ flux quanta are not bounded by a tadpole. The scalings of the fluxes are nicely consistent with keeping the number of $\mathrm{O} 6$ planes to be order one since

$$
j_{O 6} \sim e^{-\phi} m^{2} \rho^{3}\left(e^{246}-e^{136}-e^{235}+e^{145}\right),
$$

where

$$
e^{-\phi} m^{2} \rho^{3} \rightarrow \lambda^{0}=\mathcal{O}(1)
$$

Taking

$$
L_{\mathrm{AdS}}^{2}=m^{-2} \rightarrow \lambda^{2}, \quad L_{\mathrm{KK}}^{2}=\gamma^{2} \rightarrow \lambda^{2 / 3},
$$

we find scale separation

$$
L_{\mathrm{AdS}}^{2} / L_{\mathrm{KK}}^{2} \rightarrow \lambda^{4 / 3} \rightarrow \infty
$$

\section{Computation of the 6D Ricci scalar}

In this section we give the explicit computation of the Ricci scalar in terms of $\mathrm{SU}(2)$ torsion classes for the manifolds we consider in the main text. In order to do that, we use the fact that, given an $\mathrm{SU}(2)$ structure, it is always possible to embed it in an $\mathrm{SU}(3)$ structure. In this case we choose

$$
J=j-z_{R} \wedge z_{I} \quad \Omega=\omega \wedge z .
$$

Then we can express the $\mathrm{SU}(3)$ torsion classes (A.17) in terms of the $\mathrm{SU}(2)$ ones (A.18). This can be in general be very cumbersome, but it simplyfies a lot for the $\mathrm{SU}(2)$ structures compatible with the $\mathrm{O} 5 / \mathrm{O} 7$ projections, since many of the torsion classes are projected out. Comparing (A.17) and (3.8), one can show that

$$
\begin{aligned}
& W_{1}=-\frac{2}{3}\left(S_{1}+2 i S_{5}\right), \\
& W_{2}=-\frac{4}{3}\left(S_{1}-i S_{5}\right)\left(j-2 z_{R} \wedge z_{I}\right)+2 i \bar{T}_{3}, \\
& W_{3}=-2 \operatorname{Im}\left(\bar{h}_{1} z \wedge \omega\right)+2 \operatorname{Im}\left(z \wedge h^{(3)}\right), \\
& W_{4}=0=W_{5} .
\end{aligned}
$$


The expression of the Ricci scalar in terms of the $\mathrm{SU}(3)$ torsion classes is known $[56,57]$. When both $W_{4}$ and $W_{5}$ vanish the Ricci scalar is

$$
R_{6}=\frac{15}{2}\left|W_{1}\right|^{2}-\frac{1}{2}\left|W_{2}\right|^{2}-\frac{1}{2}\left|W_{3}\right|^{2} .
$$

Then using (C.2) we obtain

$$
\begin{aligned}
R_{6} & =-4\left(-3\left|e^{-A} \mu\right|^{2}+8 \operatorname{Im}\left(e^{-A} \mu h_{1}\right)+\left|h^{(3)}\right|^{2}+\left|T_{3}\right|^{2}\right), \\
& =-4\left(\left|S_{1}\right|^{2}+4\left|S_{5}\right|^{2}+\operatorname{Im}\left(S_{5} \bar{S}_{1}\right)+\left|T_{2}\right|^{2}+\left|T_{3}\right|^{2}\right) .
\end{aligned}
$$

Also note that in sourceless solution, one has (using (3.15))

$$
R_{6}=10\left|e^{-A} \mu\right|^{2}+32\left|h_{1}\right|^{2}>0 .
$$

We recover the fact that the manifold must have a positive internal curvature in order to have sourceless SUSY solution.

Open Access. This article is distributed under the terms of the Creative Commons Attribution License which permits any use, distribution and reproduction in any medium, provided the original author(s) and source are credited.

\section{References}

[1] S. Kachru, R. Kallosh, A.D. Linde and S.P. Trivedi, De Sitter vacua in string theory, Phys. Rev. D 68 (2003) 046005 [hep-th/0301240] [INSPIRE].

[2] V. Balasubramanian, P. Berglund, J.P. Conlon and F. Quevedo, Systematics of moduli stabilisation in Calabi-Yau flux compactifications, JHEP 03 (2005) 007 [hep-th/0502058] [INSPIRE].

[3] J. Polchinski and E. Silverstein, Dual purpose landscaping tools: small extra dimensions in $A d S / C F T$, arXiv:0908.0756 [INSPIRE].

[4] J.-P. Derendinger, C. Kounnas, P.M. Petropoulos and F. Zwirner, Superpotentials in IIA compactifications with general fluxes, Nucl. Phys. B 715 (2005) 211 [hep-th/0411276] [INSPIRE].

[5] K. Behrndt and M. Cvetič, General $N=1$ supersymmetric flux vacua of (massive) type IIA string theory, Phys. Rev. Lett. 95 (2005) 021601 [hep-th/0403049] [INSPIRE].

[6] O. DeWolfe, A. Giryavets, S. Kachru and W. Taylor, Type IIA moduli stabilization, JHEP 07 (2005) 066 [hep-th/0505160] [INSPIRE].

[7] D. Lüst and D. Tsimpis, Supersymmetric AdS $S_{4}$ compactifications of IIA supergravity, JHEP 02 (2005) 027 [hep-th/0412250] [INSPIRE].

[8] C. Caviezel et al., The effective theory of type IIA AdS 4 compactifications on nilmanifolds and cosets, Class. Quant. Grav. 26 (2009) 025014 [arXiv:0806.3458] [INSPIRE].

[9] A. Tomasiello, New string vacua from twistor spaces, Phys. Rev. D 78 (2008) 046007 [arXiv: 0712.1396] [INSPIRE].

[10] P. Koerber and S. Körs, A landscape of non-supersymmetric AdS vacua on coset manifolds, Phys. Rev. D 81 (2010) 105006 [arXiv:1001.0003] [INSPIRE]. 
[11] D. Tsimpis, Supersymmetric AdS vacua and separation of scales, JHEP 08 (2012) 142 [arXiv: 1206.5900] [INSPIRE].

[12] J. McOrist and S. Sethi, M-theory and type IIA flux compactifications, JHEP 12 (2012) 122 [arXiv: 1208.0261] [INSPIRE].

[13] F. Saracco and A. Tomasiello, Localized O6-plane solutions with Romans mass, JHEP 07 (2012) 077 [arXiv:1201.5378] [INSPIRE].

[14] K. Dasgupta, G. Rajesh and S. Sethi, M theory, orientifolds and G-flux, JHEP 08 (1999) 023 [hep-th/9908088] [INSPIRE].

[15] S.B. Giddings, S. Kachru and J. Polchinski, Hierarchies from fluxes in string compactifications, Phys. Rev. D 66 (2002) 106006 [hep-th/0105097] [INSPIRE].

[16] M. Graña, R. Minasian, M. Petrini and A. Tomasiello, A scan for new $N=1$ vacua on twisted tori, JHEP 05 (2007) 031 [hep-th/0609124] [INSPIRE].

[17] J. Blaback et al., Smeared versus localised sources in flux compactifications, JHEP 12 (2010) 043 [arXiv:1009.1877] [INSPIRE].

[18] J. Blaback, B. Janssen, T. Van Riet and B. Vercnocke, Fractional branes, warped compactifications and backreacted orientifold planes, JHEP 10 (2012) 139 [arXiv: 1207.0814] [INSPIRE].

[19] P. Koerber and L. Martucci, From ten to four and back again: how to generalize the geometry, JHEP 08 (2007) 059 [arXiv:0707.1038] [INSPIRE].

[20] G. Shiu, G. Torroba, B. Underwood and M.R. Douglas, Dynamics of warped flux compactifications, JHEP 06 (2008) 024 [arXiv:0803.3068] [INSPIRE].

[21] A.R. Frey, G. Torroba, B. Underwood and M.R. Douglas, The universal Kähler modulus in warped compactifications, JHEP 01 (2009) 036 [arXiv:0810.5768] [INSPIRE].

[22] L. Martucci, On moduli and effective theory of $N=1$ warped flux compactifications, JHEP 05 (2009) 027 [arXiv:0902.4031] [INSPIRE].

[23] A.R. Frey and J. Roberts, The dimensional reduction and Kähler metric of forms in flux and warping, JHEP 10 (2013) 021 [arXiv: 1308.0323] [INSPIRE].

[24] T. Banks and K. van den Broek, Massive IIA flux compactifications and U-dualities, JHEP 03 (2007) 068 [hep-th/0611185] [INSPIRE].

[25] E. Bergshoeff, Y. Lozano and T. Ortín, Massive branes, Nucl. Phys. B 518 (1998) 363 [hep-th/9712115] [INSPIRE].

[26] O. Aharony, D. Jafferis, A. Tomasiello and A. Zaffaroni, Massive type IIA string theory cannot be strongly coupled, JHEP 11 (2010) 047 [arXiv:1007.2451] [INSPIRE].

[27] K. Copsey, The instability of orientifolds and the end of the (string) landscape as we know it, arXiv:1303.4791 [INSPIRE].

[28] C. Caviezel, T. Wrase and M. Zagermann, Moduli stabilization and cosmology of type IIB on $\mathrm{SU}(2)$-structure orientifolds, JHEP 04 (2010) 011 [arXiv:0912.3287] [INSPIRE].

[29] D. Lüst and D. Tsimpis, Classes of AdS type IIA/IIB compactifications with $\mathrm{SU}(3) \times \mathrm{SU}(3)$ structure, JHEP 04 (2009) 111 [arXiv:0901.4474] [INSPIRE].

[30] M. Graña, R. Minasian, M. Petrini and A. Tomasiello, Supersymmetric backgrounds from generalized Calabi-Yau manifolds, JHEP 08 (2004) 046 [hep-th/0406137] [INSPIRE]. 
[31] M. Graña, R. Minasian, M. Petrini and A. Tomasiello, Generalized structures of $N=1$ vacua, JHEP 11 (2005) 020 [hep-th/0505212] [INSPIRE].

[32] B.S. Acharya, F. Benini and R. Valandro, Fixing moduli in exact type IIA flux vacua, JHEP 02 (2007) 018 [hep-th/0607223] [INSPIRE].

[33] P. Koerber, D. Lüst and D. Tsimpis, Type IIA AdS 4 compactifications on cosets, interpolations and domain walls, JHEP 07 (2008) 017 [arXiv:0804.0614] [INSPIRE].

[34] N. Hitchin, Generalized Calabi-Yau manifolds, Quart. J. Math. Oxford Ser. 54 (2003) 281 [math/0209099] [INSPIRE].

[35] M. Gualtieri, Generalized complex geometry, math/0401221 [INSPIRE].

[36] U.H. Danielsson et al., De Sitter hunting in a classical landscape, Fortsch. Phys. 59 (2011) 897 [arXiv:1103.4858] [INSPIRE].

[37] K. Behrndt, M. Cvetič and P. Gao, General type IIB fluxes with SU(3) structures, Nucl. Phys. B 721 (2005) 287 [hep-th/0502154] [INSPIRE].

[38] P. Koerber and L. Martucci, D-branes on AdS flux compactifications, JHEP 01 (2008) 047 [arXiv:0710.5530] [INSPIRE].

[39] J.P. Gauntlett, D. Martelli, S. Pakis and D. Waldram, G structures and wrapped NS5-branes, Commun. Math. Phys. 247 (2004) 421 [hep-th/0205050] [INSPIRE].

[40] J.P. Gauntlett, D. Martelli and D. Waldram, Superstrings with intrinsic torsion, Phys. Rev. D 69 (2004) 086002 [hep-th/0302158] [INSPIRE].

[41] G. Dall'Agata and N. Prezas, $N=1$ geometries for $M$-theory and type IIA strings with fluxes, Phys. Rev. D 69 (2004) 066004 [hep-th/0311146] [INSPIRE].

[42] P. Koerber and D. Tsimpis, Supersymmetric sources, integrability and generalized-structure compactifications, JHEP 08 (2007) 082 [arXiv:0706.1244] [INSPIRE].

[43] M.P. Hertzberg, S. Kachru, W. Taylor and M. Tegmark, Inflationary constraints on type IIA string theory, JHEP 12 (2007) 095 [arXiv:0711.2512] [INSPIRE].

[44] U.H. Danielsson, S.S. Haque, G. Shiu and T. Van Riet, Towards classical de Sitter solutions in string theory, JHEP 09 (2009) 114 [arXiv: 0907. 2041] [INSPIRE].

[45] J.M. Maldacena and C. Núnez, Supergravity description of field theories on curved manifolds and a no go theorem, Int. J. Mod. Phys. A 16 (2001) 822 [hep-th/0007018] [InSPIRE].

[46] M.R. Douglas and R. Kallosh, Compactification on negatively curved manifolds, JHEP 06 (2010) 004 [arXiv: 1001.4008] [INSPIRE].

[47] P.G. Freund and M.A. Rubin, Dynamics of dimensional reduction, Phys. Lett. B 97 (1980) 233 [INSPIRE].

[48] M. Larfors, D. Lüst and D. Tsimpis, Flux compactification on smooth, compact three-dimensional toric varieties, JHEP 07 (2010) 073 [arXiv:1005.2194] [INSPIRE].

[49] S.P. Dabholkar, Toric manifolds for flux compactification, arXiv:1301.2623 [INSPIRE].

[50] G.R. Cavalcanti and M. Gualtieri, Generalized complex structures on nilmanifolds, J. Symplectic Geom. 2 (2004) 393 [math.DG/0404451].

[51] U.H. Danielsson, P. Koerber and T. Van Riet, Universal de Sitter solutions at tree-level, JHEP 05 (2010) 090 [arXiv: 1003.3590] [INSPIRE]. 
[52] D. Andriot, E. Goi, R. Minasian and M. Petrini, Supersymmetry breaking branes on solvmanifolds and de Sitter vacua in string theory, JHEP 05 (2011) 028 [arXiv:1003.3774] [INSPIRE].

[53] E. Silverstein, Simple de Sitter solutions, Phys. Rev. D 77 (2008) 106006 [arXiv:0712.1196] [INSPIRE].

[54] S.S. Haque, G. Shiu, B. Underwood and T. Van Riet, Minimal simple de Sitter solutions, Phys. Rev. D 79 (2009) 086005 [arXiv:0810.5328] [InSPIRE].

[55] A. Barranco, J. Gaillard, N.T. Macpherson, C. Núñez and D.C. Thompson, G-structures and flavouring non-Abelian T-duality, JHEP 08 (2013) 018 [arXiv: 1305.7229] [INSPIRE].

[56] T. Ali and G.B. Cleaver, The Ricci curvature of half-flat manifolds, JHEP 05 (2007) 009 [hep-th/0612171] [INSPIRE].

[57] L. Bedulli and L. Vezzoni, The Ricci tensor of SU(3)-manifolds, J. Geom. Phys. 57 (2007) 1125 [math.DG/0606786]. 